\title{
A Novel Translational Model of Spinal Cord Injury in Nonhuman Primate
}

\author{
Marine Le Corre ${ }^{1,2} \cdot$ Harun N. Noristani ${ }^{1,3} \cdot$ Nadine Mestre-Frances ${ }^{4}$. \\ Guillaume P. Saint-Martin ${ }^{3,5}$. Christophe Coillot ${ }^{5}$. Christophe Goze-Bac ${ }^{5}$. \\ Nicolas Lonjon ${ }^{2,3}$. Florence E. Perrin ${ }^{1,3}$ (I)
}

Published online: 27 November 2017

(C) The American Society for Experimental NeuroTherapeutics, Inc. 2017

\begin{abstract}
Spinal cord injuries (SCI) lead to major disabilities affecting $>2.5$ million people worldwide. Major shortcomings in clinical translation result from multiple factors, including species differences, development of moderately predictive animal models, and differences in methodologies between preclinical and clinical studies. To overcome these obstacles, we first conducted a comparative neuroanatomical analysis of the spinal cord between mice, Microcebus murinus (a nonhuman primate), and humans. Next, we developed and characterized a new model of lateral spinal cord hemisection in M. murinus. Over a 3month period after SCI, we carried out a detailed, longitudinal, behavioral follow-up associated with in vivo magnetic resonance imaging $\left({ }^{1} \mathrm{H}-\mathrm{MRI}\right)$ monitoring. Then, we compared lesion extension and tissue alteration using 3 methods: in vivo ${ }^{1} \mathrm{H}-\mathrm{MRI}$, ex vivo ${ }^{1} \mathrm{H}-\mathrm{MRI}$, and classical histology. The general organization and glial cell distribution/morphology in the spinal cord of M. murinus closely resembles that of humans. Animals assessed at different stages following lateral hemisection of the spinal cord presented specific motor deficits and spinal cord tissue alterations. We also found a close correlation between ${ }^{1} \mathrm{H}-\mathrm{MRI}$ signal and microglia reactivity and/or associated post-trauma phenomena. Spinal cord hemisection in M. murinus provides a reliable new nonhuman primate model that can be used to promote translational research on SCI and represents a novel and more affordable alternative to larger primates.
\end{abstract}

Keywords Spinal cord injury $\cdot$ non human primate $\cdot$ behavior $\cdot$ MRI $\cdot$ histopathology $\cdot$ microglia

\section{Introduction}

Marine Le Corre, Harun N. Noristani, Nicolas Lonjon and Florence E. Perrin contributed equally to this work.

Electronic supplementary material The online version of this article (https://doi.org/10.1007/s13311-017-0589-9) contains supplementary material, which is available to authorized users.

Florence E. Perrin

florence.perrin@inserm.fr

1 INSERM U1051, Rue Augustin Fliche, F-34095 Montpellier Cedex 5 , France

2 CHRU Montpellier, Gui de Chauliac Hospital, F-34095 Montpellier, France

3 INSERM U1198, University of Montpellier, EPHE, Place Eugène Bataillon CC105, F-34095 Montpellier, France

4 INSERM U1198, University of Montpellier, EPHE, PSL Research University, Place Eugène Bataillon CC105,

F-34095 Montpellier, France

5 CNRS UMR 5221, University of Montpellier, Place Eugène Bataillon, F-34095 Montpellier, France
The number of people affected by spinal cord injuries (SCI) ranges from 2.5 to 4 million worldwide $[1,2]$. SCI is not restricted to traumatic events; indeed, nontraumatic SCI occurs in diseases such as degenerative disk disease, infection, and tumor. Traumatic SCI predominantly affect males and young individuals; however, the proportion of older persons affected by SCI is also increasing in parallel with the aging world population [3]. In traumatic SCI a mechanical trauma induces the primary injury, which is characterized by focal cellular, vascular, and blood-spinal cord barrier damages. These events induce and propagate secondary injuries such as infiltration of blood-derived inflammatory cells, ischemia, edema inflammation, glial scar and cystic cavities formation, excitotoxicity, and cellular demise. Subsequently, secondary damages include deposition of extracellular matrix protein at the lesion epicenter [3, 4]. These numerous secondary events following SCI can harvest greater impairment than the primary mechanical insult. Some sensory and motor functions caudal to the injury site remain partially intact after incomplete $\mathrm{SCI}$, whereas a total loss of function is induced by a complete lesion of the spinal cord. 
Many factors account for the shortage of findings being translated from bench to bedside, including species differences, the unavailability of predictive animal models, and differences in the methodologies used in preclinical animal models and human studies. Significant differences in the neuroanatomical organization of motor and sensory systems, as well as neurophysiological variations between rodents and primates [5] may explain why responses to SCI vary considerably between species [6]. Important variations in corticospinal projections result in species-specific differences in sensorimotor behavior [7-9]. Indeed, the distinct corticospinal tract (CST) architecture in primates could explain their superior functional recovery after lateralized injuries versus rodents [6], as well as the spontaneous functional improvements observed early after injury in humans with partial lesions of the spinal cord [10]. These studies emphasize the crucial need for nonhuman primate (NHP) SCI models that are more predictive and that may enhance translational research [11-13].

As a result of SCI, a glial scar composed of activated astrocytes and microglia forms an inhibitory barrier to spontaneous axonal regeneration [14]. In humans, data on the composition of the scar tissue are scarce. Postmortem analysis of human spinal cords taken from patients that had died between 2 days and 30 years after traumatic SCI speaks in favor of a predominant inhibitory role of the astroglial compartment in axonal regeneration [15]. At the lesion epicenter early after injury (between 2 and 11 days) glial fibrillary acidic protein (GFAP) immunoreactivity revealed a total loss of astrocytes [15], and by 10 and 11 days after lesion macrophages are found [4]. In the intermediate zone, which includes the borders of the lesion site, a dramatic loss of the astroglial framework is also observed up to 10 and 11 days postinjury before the initial signs of astrocytic reactivity is detected. In the perilesional area, the astroglial framework remains intact and reactive astrocytes extend their processes in the white matter. By 24 days following insult, Schwann cell migration into the lesion site is reported [4] but not in the intermediate zone [15], where the first signs of astrocytic scar formation are detected $[4,15]$. By 4 months after SCI 2 distinct regions are seen in the lesion area, the first predominantly containing infiltrated Schwann cells (which is further subdivided into areas rich in extracellular matrix protein or in neuromas [4]), and the second region dominated by astrocytes [15]. From 4 months post-SCI, in the area with densely packed GFAP-positive astroglial cells, thin neurofilament-positive axons are also observed. It has also been reported in NHP that SCI-induced astrogliosis in areas undergoing Wallerian degeneration is moderate with only a slight increase in GFAP expression at 4 months postinjury, which returns to normal and stabilizes within 1 year after the traumatism [16]. This late astrocytic reaction is followed by the long-term deposition of a dense anisotropic GFAP-positive matrix in degenerating areas and in the glial scar [17].
In Macaca fascicularis, $1 \mathrm{~h}$ after spinal cord compression there are marked increase in macrophage-related protein 8 , matrix metallopeptidase 9 , ionized calcium-binding adapter molecule 1 (IBA-1), and inducible nitric oxide synthase in microglia/macrophages at the lesion's epicenter [18]. Between 7 and 30 days after spinal cord hemisection in M. fascicularis, microglia/macrophage activation is evident not only at the lesion site, but also in the spared contralateral hemi-spinal cord and in the peri-lesional area; however, in contrast to rodents, no substantial astrogliosis is evident at the lesion's edge [19]. In fact, no reactive astrocytes have been found within areas of Wallerian degeneration up to 4 weeks following spinal cord hemisection in M. fascicularis; moreover, there is a clear lack of amoeboid microglia/macrophages, suggesting either an incomplete or delayed gliosis [16]. Gene expression analysis in marmosets also revealed a delayed glial scar formation and a prolonged inflammatory response versus rodents, further supporting temporal differences in glial response after injury among species [17]. In the lesional penumbra, no strong astrocytic glial scars have been observed 6 months after spinal cord contusion in rhesus macaques, but strong GFAP immunoreactivity is present in the spared white matter [20].

Although macaques (M. fascicularis) are being used as NHP models for SCI, there are several limitations associated with their use, including their large body size (length 38.5$64.8 \mathrm{~cm}$; weight $2500-8300 \mathrm{~g}$ ), delayed maturity (3-5 years), low reproductive output (1 offspring), and long interbirth intervals (18 months) that altogether are critical for building sufficient colony size [21]. To address some of these issues, smaller primates, such as common marmosets (Callithrix jacchus), are considered more suitable as a NHP model for SCI with relatively smaller body size (length $20-35 \mathrm{~cm}$; weight 200-600 g), shorter developmental period (18 months), better reproductive output (1-2 offspring), and shorter interbirth intervals (6 months; see Table 1) [22]. However, both $M$. fascicularis and $C$. jacchus carry and can transmit serious zoonotic diseases, including hepatitis B [23], herpes virus [24, 25], and tuberculosis [26, 27]. Furthermore, high maintenance costs can limit their widespread application as animal models for central nervous system trauma.

As another NHP, the gray mouse lemur (Microcebus murinus) not only have common genetic, physiological, and neuroanatomical similarities with humans, but they also resemble the heterogeneity present among human population [28]. In comparison with other NHPs, M. murinus has several advantages, including 1) small body size (length $\sim 12 \mathrm{~cm}$; weight $\sim 60-120 \mathrm{~g}$ ); 2 ) rapid maturity (1 year); and 3) higher fecundity (gestation 2 months; $1-3$ offspring per litter) [29]. In addition, to the best of our knowledge, there are no reports of M. murinus carrying any serious zoonotic diseases. Altogether, these features make M. murinus better suited for experimental manipulation such as SCI. Despite these obvious 
Table 1 Major characteristics of nonhuman primates used in the spinal cord injury field

\begin{tabular}{llll}
\hline Characteristic & $\begin{array}{l}\text { Long-tailed macaque } \\
\text { (Macaca Fascicularis) }\end{array}$ & $\begin{array}{l}\text { Common marmoset } \\
\text { (Callithrix jacchus) }\end{array}$ & $\begin{array}{l}\text { Gray mouse lemur } \\
\text { (Microcebus murinus) }\end{array}$ \\
\hline Body length $(\mathrm{cm})$ & $38.5-64.8$ & $20-35$ & 12 \\
Body weight $(\mathrm{g})$ & $2500-8300$ & $200-600$ & $60-120$ \\
Developmental period (months) & $36-60$ & 18 & $<12$ \\
Number of offspring & 1 & $1-2$ & $1-3$ \\
Interbirth intervals (months) & 18 & 6 & 2 \\
Carrier of zoonotic diseases & Yes & Yes & No \\
\hline
\end{tabular}

advantages, no study has used $M$. murinus as a preclinical animal model for SCI.

Noninvasive longitudinal studies are mandatory for studying lesion evolution in vivo. Magnetic resonance imaging (MRI) is a widely used approach to monitor SCI in humans, and recent MRI studies, including our own [30, 31], have been used effectively for SCI follow-up in rodents.

Here, we describe glial cell distribution/morphology in the spinal cord of $M$. murinus versus that of mice and humans. We then used this species to develop a new model of lateral spinal cord hemisection and carried out a detailed behavioral assessment before and after SCI. We also used noninvasive quantitative ${ }^{1} \mathrm{H}$-MRI to follow lesion evolution up to 3 months postlesion. We then correlated in vivo ${ }^{1} \mathrm{H}-\mathrm{MRI}$ findings at 3 months postinjury with postmortem high-resolution ex vivo ${ }^{1} \mathrm{H}-\mathrm{MRI}$ images and detailed histology. Our findings indicate that glial cell distribution/morphology in the spinal cord of M. murinus closely resembles that of humans. Lateral hemisection induced behavioral deficits that can be quantitatively assessed at different stages postinjury. Interestingly, we establish for the first time a coincidence between cellular modification, i.e., microglial reactivity (and/or associated phenomena), and distinct ${ }^{1} \mathrm{H}$-MRI signal after injury. These data demonstrate that lateral hemisection injury in M. murinus constitutes a novel and more affordable NHP model that can be used to improve translational research on SCI.

\section{Methods}

Experimental procedures followed the French and European legislative, administrative, and statutory measures for animal experimentation (European Union Directive 2010/63 of the European Parliament and Council). The study was approved by the "Direction des Services Vétérinaires de l'Hérault" and the "Ministère de l'éducation nationale, de l'enseignement supérieur et de la recherche, ethic committee N`36 ", France (authorization number CEEA-LR-12142).

Human spinal cords were obtained from the New York Brain Bank, Taub Institute, Columbia University (NYBB),
New York, NY, USA. All donors had given their written consent.

\section{SCI}

\section{Animals}

Five adult male gray mouse lemurs ( $M$. murinus, 2 years old) were used. All $M$. murinus were born and bred in the animal facility at CECEMA, University of Montpellier, France. Two M. murinus were used to set up either surgical, behavioral, or in vivo ${ }^{1} \mathrm{H}$-MRI protocols and did not go through all in vivo experiments at the exact same time points; thus, only 3 animals are presented in the behavioral and in vivo ${ }^{1} \mathrm{H}-\mathrm{MRI}$ data. We also obtained 3 spinal cords from noninjured adult M. murinus for comparative histological experiments with mice and humans, these lemurs had not been perfused with paraformaldehyde (PFA). Animals were housed together in cages $(2 \mathrm{~m} \times 1 \mathrm{~m} \times 1 \mathrm{~m}, 3$ lemurs per cage) until surgery, then separately $(60 \mathrm{~cm} \times 60 \mathrm{~cm} \times 50 \mathrm{~cm})$ for 1 week following injury before they were returned to their original cages. All cages were equipped with wooden nests. Animals were kept at standardized temperature $\left(24-26^{\circ} \mathrm{C}\right)$ and relative humidity (55\%) and fed with fresh fruit and a mixture of cereal, milk, and eggs, prepared daily. Water and food were given ad libitum. Animals were given mealworms $24 \mathrm{~h}$ after surgery to increase their protein intake.

\section{Lateral Hemisection}

Atropine (0.4-0.6 mg/kg; Aguettant, Lyon, France) was administered subcutaneously $15 \mathrm{~min}$ prior to surgery to inhibit salivary and bronchial secretions, as well as vagal stimulation. Food was withdrawn $12 \mathrm{~h}$ prior to surgery, anesthesia was induced with $3 \%$ to $4 \%$ isoflurane (Vetflurane; Virbac, Carros, France) and maintained with a mixture of $1 \%$ to $2 \%$ isoflurane and $1 \mathrm{l} / \mathrm{min}$ oxygen flow rate throughout the surgery. Eye gel was applied to the cornea from the beginning of the anesthesia. The skin was shaved and cleaned with an antiseptic (Vetadine; Bayer, Pymble, Australia). The skin and muscles overlying the thoracolumbar segment were cut along 
the back midline and a laminectomy of the posterior arch of the lumbar vertebra 1 (Fig. S1A, B, arrows) was carried out. Lateral hemisection of the spinal cord was done under a microscope using a micro knife (10315-12; Fine Science Tools, Foster City, CA, USA). The surgical area was cleaned with an antiseptic (Vetadine; Bayer). Muscles and skin were sutured and animals were left to recover on a temperaturecontrolled pad. M. murinus were observed twice daily. Signs of pain or distress were carefully observed. These signs included a refusal to eat and drink, a bent posture, an absence or decrease in grooming activity, and self-mutilation (biting, hair removal, shots on the face and eyes). Bladder function was controlled and, if necessary, emptied manually. Animals were given analgesia (bupremorphine, $0.01 \mathrm{mg} / \mathrm{kg} / \mathrm{day}, \mathrm{i} . \mathrm{m}$.) and antibiotic [enrofloxacin ( $5 \mathrm{mg} / \mathrm{kg} /$ day, s.c.)] for $48 \mathrm{~h}$ after SCI. One animal developed self-biting and received bupremorphine and enrofloxacin for 1 week after starting of self-injury. Body weights were measured daily until stabilization, then once a week. Animals were kept for 3 months postlesion.

\section{Behavioral Analysis}

Behavioral assessments started with training periods $(10,5$, and 2 days prior to surgery) followed by tests at 1,3 , and 7 days postsurgery and then once a week until 3 months after lesion.

\section{Open Field Test}

Spontaneous functional motor activity was evaluated by 2 independent experimenters; each hindlimb was observed and scored separately. Animals were placed in an empty Plexiglass test arena $(50 \times 60 \mathrm{~cm})$ and observed for at least $10 \mathrm{~min}$. We adapted motor behavioral scoring previously used in rats $[32,33]$. Scores were as follows: $0=$ no movement of the hindlimb; 1 = barely perceptible hindlimb movement; 2 = frequent hindlimb movement, no weightbearing; 3 = partial weight support, no walking; $4=$ total weight support, walking with a major deficit; 5 = walking with mild deficits; $6=$ normal walking.

\section{Grip Test}

The ability to grip was evaluated by 2 independent experimenters using an inclined grip test $\left(60^{\circ}, 60 \mathrm{~cm}\right)$ that allowed animals to reach their nest. Animals were placed facing the experimenters and each hindlimb was scored. Scores were as follows: $0=$ no attempt to grip the bar, no movement of the toes; 1 = partial grip, attempt to grip but consistent foot faults, foot slips off the bar, and/or accurate foot placement on bar but toes do not grip; and 2 = normal grip of the toes on the bar (see Fig. 3C, zoom all toes).

\section{CatWalk}

CatWalk (Noldus, Wageningen, The Netherlands) acquisition was done immediately after open-field and grid tests. Animals walked toward their nest over a 1 meter-long glass walkway with a fluorescently illuminated floor to record footprints. A minimum of 5 uninterrupted walks were collected per animal at given time points. Data were analyzed using CatWalk 7.1 software (Noldus) and CatMerge (InnovationNet, Tiranges, France). Several parameters were quantified, related either to individual footprints (e.g., width and length of a complete paw print, pressure exerted by a paw, duration of contact of a paw with the glass plate, area of the print at max contact) or to the position of footprints in a step cycle (stride length, base of support, relative print position). For example, "base of support" corresponds to the average width between either the front or the hindpaws; "stand" relates to the duration of contact between the paw and the glass plate in a step cycle; "swing" is the duration of no contact between the paw and the glass plate in a step cycle; and "regularity index" expresses the number of normal step sequence patterns relative to the total number of paw placements.

\section{${ }^{1}$ H-MRI}

\section{In vivo ${ }^{1} \mathrm{H}-\mathrm{MRI}$}

${ }^{1} \mathrm{H}-\mathrm{MRI}$ acquisition was done using a 9.4 Tesla apparatus (Agilent Varian 9.4/160/ASR; Agilent, Santa Clara, CA, USA) equipped with a MAGNEX TS1276D, a Quadrature Volume Coil 400MHz RF72 (Rapid Biomedical, Rimpar, Germany), and associated with a VnmrJ Imaging acquisition system (Agilent).

M. murinus were anesthetized as previously described using a MR-compatible anesthetic machine and animal holder system. Respiration and body temperature were continuously monitored using the MR-compatible Small Animal Monitoring and Gating System. Respiration was maintained around 30 breaths/min. Longitudinal analysis was done at the following time points: $48 \mathrm{~h}$, and 1, 4, and 12 weeks after lesion.

Multiple echo multislice acquisition protocol was used to obtain axial images using the following parameters: repetition time $(\mathrm{TR})=2500 \mathrm{~ms}$; echo time $(\mathrm{TE})=12.63 \mathrm{~ms}$; echo number $(\mathrm{NE})=2$; average $(\mathrm{AVG})=2$; field of view $(\mathrm{FOV})=$ $60 \mathrm{~mm} * 60 \mathrm{~mm} ; 35$ slices; thickness $=0.8 \mathrm{~mm}$; gap $=0 \mathrm{~mm}$; acquisition matrix $\left(\mathrm{N}_{\mathrm{READ}} * \mathrm{~N}_{\mathrm{PHASE}}\right)=512 * 512$. Image acquisition was synchronized with respiration to reduce motion artefacts; the scanning time was approximately $1 \mathrm{~h}$. 


\section{Ex vivo ${ }^{1} \mathrm{H}-\mathrm{MRI}$}

Three months after hemisection, M. murinus were anesthetized with ketamine and perfused intracardially with cold $0.1 \mathrm{M}$ phosphate saline buffer (PBS) followed by cold 4\% PFA (pH 7.2; Sigma Aldrich, St. Louis, MO, USA), the spinal cords were dissected and postfixed for $2 \mathrm{~h}$ in $4 \%$ PFA. To enhance contrast, spinal cords were then incubated for $48 \mathrm{~h}$ in gadolinium $(1: 100$ in $0.1 \mathrm{M}$ PBS). Just prior to ex vivo ${ }^{l} \mathrm{H}$-MRI, tissues were placed in Fluorinert FC-40 liquid in a 5-mm-diameter glass tube, as previously described [30, 31, 34]. Quadrature volume coil 400 MHz RF43 (Rapid Biomedical) was used. Axial images were obtained with multiple echo multislice acquisition protocol using the following parameters: $\mathrm{TR}=1155 \mathrm{~ms}$; TE $=$ $13.47 \mathrm{~ms} ; \mathrm{NE}=1 ; \mathrm{AVG}=180 ; \mathrm{FOV}=10 \mathrm{~mm} * 10 \mathrm{~mm} ; 60$ slices, thickness $=0.6 \mathrm{~mm}$; gap $=0 \mathrm{~mm}$; acquisition matrix $=$ $256 * 256$. Scanning time was approximately $15 \mathrm{~h}$. MRI segmentation was done using Myrian Software.

Immediately after ex vivo ${ }^{1} \mathrm{H}-\mathrm{MRI}$ acquisition, spinal cords were rinsed in $0.1 \mathrm{M}$ PBS, cryoprotected in $30 \%$ sucrose, embedded in Tissue Tek (Sakura, Alphen aan den Rijn, The Netherlands), frozen and kept at $-80^{\circ} \mathrm{C}$. Serial $14-\mu \mathrm{m}-$ thick axial spinal cord cryosections were collected on Superfrost Plus slides.

\section{Neuroanatomical Comparison Between Species}

\section{Animal Spinal Cord Samples}

Spinal cords from 3 noninjured M. murinus were used as a control. Additionally, eight 3-month-old female mice were used, 3 Swiss and 5 C57BL/6. Mice were anesthetized, perfused intracardially as before, and a series of 14- $\mu \mathrm{m}$-thick axial thoracic and lumbar spinal cord cryosections were collected, as described above.

\section{Human Spinal Cord Samples}

Human lower thoracic and lumbar (T11-L2) spinal cords were obtained from 15 controls (11 males and 4 females, mean age 54 years, from the New York Brain Bank, Taub Institute, Columbia University, New York, NY, USA). The cause of death [road accident or fall $(n=5)$, myocardial infarction ( $n$ $=3)$, stroke $(n=3)$, and aneurysm rupture $(n=4)$ ] can almost exclude the existence of motor impairments prior to death. Twenty-two- $\mu \mathrm{m}$-thick cryosections of lower thoracic/lumbar segments were cut and mounted on Superfrost Plus slides, as described above.

\section{Histology}

Toluidine blue staining was used to analyze the overall neuroanatomical structure (all species). In the undamaged spinal cord, toluidine blue staining predominantly labels neuron in the gray matter. Slides were placed for $3 \mathrm{~min}$ in $0.1 \%$ toluidine blue solution followed by $10 \mathrm{~s}$ in $100 \%$ ethanol and 2 repetitions of $3 \mathrm{~min}$ in xylene. All slides were cover-slipped using Entellan (Merck, Darmstadt, Germany). Morphometric brightfield photographs were obtained and analyzed using NanoZoomer scanner (NanoZoomer Digital Pathology System and NDP view software, Hamamatsu, Japan).

\section{Antibodies}

Primary antibodies used included rabbit anti-GFAP (1:500; Dako, Glostrup, Denmark) and rabbit anti-IBA-1 (1:200; Wako Pure Chemical Industries, Tokyo, Japan).

\section{Histological Analysis of $\mathrm{SCl}$ in Microcebus murinus}

\section{Lesion Extension and Volume}

Toluidine blue staining was done as described above and used to quantify lesion extension after injury. The lesion site was clearly identifiable at low magnification with numerous small toluidine blue-positive profiles surrounding the damaged/ disorganized tissue. The lesion area was measured as percentage of the total surface area in each spinal cord section. Similarly, areas of undamaged tissue within the white and gray matters were measured (given in $\mu \mathrm{m}^{2}$ ) to estimate lesion extension. For each M. murinus we analyzed at least fifty $14-\mu \mathrm{m}$-thick serial sections throughout the lesion segment of the spinal cord at $210-\mu \mathrm{m}$ intervals ( 1 out of every 15 sections was quantified). Lesion volume was measured per section by multiplying the lesion surface area by the section thickness. Measured volume was then multiplied by 14 (1 section stained each 15 sections), then all volumes obtained were summed for a final estimation of lesion volume in cubic millimeters.

\section{Immunohistochemistry}

Spinal cord sections were washed twice in $0.1 \mathrm{M}$ PBS, then treated for $30 \mathrm{~min}$ in $0.1 \mathrm{M} \mathrm{PBS}+$ lysine $(20 \mathrm{mM}, \mathrm{pH} 7.2)$ and $15 \mathrm{~min}$ in hydrogen peroxide $\left(\mathrm{H}_{2} \mathrm{O}_{2} ; 1 \%\right)$. Sections were blocked for $2 \mathrm{~h}$ with $0.1 \mathrm{M}$ PBS containing $1 \%$ bovine serum albumin and Triton X-100 (0.1\%), then incubated for $24 \mathrm{~h}$ at room temperature (mice) and $48 \mathrm{~h}$ at $4^{\circ} \mathrm{C}(M$. murinus and humans) with the primary antibody. Slides were rinsed with $0.1 \mathrm{M}$ PBS for $30 \mathrm{~min}$ and incubated in 1:1000 dilutions of the corresponding secondary antibody linked to horseradish peroxidase (Jackson Immunoresearch, Newmarket, UK) for $1 \mathrm{~h}$ at room temperature. Slides were rinsed with 0.1 M PBS for $30 \mathrm{~min}$. The product of the peroxidase reaction was visualized by incubating slides in a solution containing $0.022 \% 3,3^{\prime}$ diaminobenzidine and $0.003 \% \mathrm{H}_{2} \mathrm{O}_{2}$ for $30 \mathrm{~min}$. The reaction was stopped by rinsing the sections in $0.1 \mathrm{M}$ PBS for $15 \mathrm{~min}$. 
Slides were then dehydrated in increasing concentrations of ethanol and, finally, xylene. Cover slips were applied using Entellan (Merck). Negative controls were used in which the primary antibody was omitted.

Morphometric brightfield photographs were obtained using constant exposure time and analyzed with NanoZoomer RS slide scanner (NanoZoomer Digital Pathology System and NDP view software; Hamamatsu). To determine SCI-induced changes in astrogliosis (GFAP) and microglia/macrophage (IBA-1) responses in M. murinus, the mean optical density (OD) was measured along the spinal cord at 3 months after spinal cord hemisection using ImageJ (National Institutes of Health, Bethesda, MD, USA), as described previously [35]. OD is a sensitive and reliable technique with which to measure the expression of a given signal and to detect changes caused by different experimental conditions. Digital images for all sections were exported as RGB using identical exposure settings. To avoid variation in staining intensity between different slides or animals, all immunostainings for a given antibody were done in parallel. GFAP and IBA-1 intensities were analyzed in at least $50 \mathrm{sec}-$ tions throughout the lesion segment of the spinal cord at $210-\mu \mathrm{m}$ intervals. Regions of interest (white and gray matters, dorsal funiculus) across spinal cord sections were outlined and the mean OD was measured. The lesion epicenter (section with highest damaged area) allowed determination of intensity differences both rostral and caudal to the lesion site. Background OD was subtracted from OD values of each section.

\section{Neuromuscular Junction Labelling}

Following M. murinus perfusion, the gastrocnemius-soleus-plantaris muscular complex were collected for neuromuscular junction (NMJ) labelling using the method described by Karnovsky and Roots [36]. Transverse sections $(16 \mu \mathrm{m})$ of the entire muscle complex were analyzed. NMJs were quantified in every 3 sections by a blinded experimenter.

\section{Mini-Atlas of Microcebus murinus Vertebral Column and Spinal Cord}

Computer tomography of the body of one M. murinus had been obtained using CT Discovery 750 HD (GE Healthcare, Milwaukee, WI, USA; Fig. S1). Ex vivo diffusion MRIs of uninjured M. murinus (2 years of age) were acquired using a spinal cord that had been postfixed for $48 \mathrm{~h}$ in cold 4\% PFA ( $\mathrm{pH} 7.2$; Sigma Aldrich). Just prior to ex vivo diffusion ${ }^{1} \mathrm{H}-\mathrm{MRI}$, tissues were placed in Fluorinert FC-40 liquid in a 4-mm-diameter glass tube, as previously described (Fig. S1, Video S1). MRI Ribbon Coil dedicated to SCI investigations $400 \mathrm{MHz}$ was used
[34]. The following acquisition parameters were used: diffusion-weighted axial images were acquired using 2 sequential spin-echo multislices (SEMS-dw) protocols. Acquisition parameters were as follows: delta $=6.88 \mathrm{~ms}$, $\mathrm{Gs}=10 \mathrm{G} / \mathrm{cm}$, separation $=15.05 \mathrm{~ms}(\mathrm{~b}$-value $=522.96$ $\left.\mathrm{s} / \mathrm{mm}^{2}\right), \mathrm{TR}=1577.34 \mathrm{~ms}, \mathrm{TE}=34.27 \mathrm{~ms}, \mathrm{AVG}=30$, $\mathrm{FOV}=10 \times 10$, slices $=30$, thickness $=1$, gap $=0$, acquisition matrix $=256 \times 256$. For the second sequence, the dwi parameters were repeated but without diffusion gradient applied on longitudinal axis ( $\mathrm{Gs}=0, \mathrm{~b}$-value $\approx 0$ $\mathrm{s} / \mathrm{mm}^{2}$ ). Scanning time was approximately $3 \mathrm{~h}$ per protocol.

Histological stains were done on 1 postfixed and cryoprotected spinal cord, frozen, and stored at $-80^{\circ} \mathrm{C}$. Freefloating $40-\mu \mathrm{m}$-thick cryosections of cervical, thoracic, lumbar, and sacral segments were collected and placed in $0.1 \mathrm{M}$ PBS. Prior to staining all sections were postfixed for $2 \mathrm{~h}$ in $4 \%$ PFA. Two stains, on at least 5 spinal cord sections, were done for each segment: $0.1 \%$ toluidine blue solution (incubation 10 $\mathrm{min}$ ) and cresyl violet (Nissl staining, $0.2 \%$, incubation time $20 \mathrm{~min}$ ). All sections were mounted on gelatin-coated slides, dehydrated (100\% ethanol), washed in xylene, and then cover slipped using Entellan (Merck).

\section{Artwork and Statistics}

All images were assembled using Photoshop (Adobe, San Jose, CA, USA) as the image-editing program. All statistical tests were done using GraphPad Prism version 5.03 (GraphPad Software, La Jolla, CA, USA). Significance was accepted at $p \leq 0.05$. Results are expressed as mean \pm SEM. Neuroanatomical comparison between species was done with an unpaired $t$-test. Behavior calculated by score of the hindlimb on the injured versus the noninjured side was analyzed by 2-way analysis of variance (ANOVA) with Bonferroni post-hoc tests. Behavior using CatWalk was analyzed by 1-way ANOVA with Bonferroni post-hoc tests. Lesion extension by in vivo ${ }^{1} \mathrm{H}-\mathrm{MRI}$ was analyzed by 2 way ANOVA with Bonferroni post-hoc tests. Lesion volume and histology was analyzed by paired $t$-test. GFAP and IBA-1 intensities at similar rostral and caudal distances from the lesion site and NMJ density were analyzed by paired $t$-tests.

\section{Results}

All data analyzed during this study are included in this published article and the supplementary files; raw behavioral and MRI datasets are available from the corresponding author upon reasonable request. 


\section{Neuroanatomical Characterization of Microcebus murinus Spinal Cord}

To better characterize the rachis and spinal cord of M. murinus, we first carried out a computerized tomography scan to illustrate the organization of the skeleton and, in particular, the vertebra (Fig. S1A-D). The spinal cord was then dissected (Fig. S1E) and imaged using ex vivo diffusion MRI (Fig. S1F-I, Video S1). Furthermore, we sectioned and analyzed the dissected spinal cord from uninjured M. murinus using classical histological stains, including cresyl violet (Fig. S1J-M) and toluidine blue (Fig. S1N-Q) at different rostrocaudal levels (Fig. S1N-Q). We then examined the spinal cord of M. murinus and compared the neuroanatomical features in lower thoracic/higher lumbar levels with those in mice and humans. Toluidine blue staining was used for general histology, more specifically to stain nuclei and analyze their distribution within the gray and white matters of the spinal cord (Fig. 1A-C). We observed a clear variation in the proportions of gray and white matters between the 3 species. Quantification of the surface area of both matters revealed a higher white-to-gray matter ratio in primate than in rodent spinal cords and no significant difference between M. murinus and humans (Fig. 1D-F).

We next characterized microglia and astrocytic distribution/morphology within the spinal cord in all 3 species
(Fig. 2). IBA-1 immunostaining demonstrated a heterogeneous microglial distribution, with higher density in the gray matter of all 3 species (Fig. 2A-C). Morphologically, IBA-1positive microglia in mice were ramified with small perikarya and randomly distributed thin-to-medium-sized processes in both gray (Fig. S2A, C, arrow) and white matter (Fig. 2D, arrow). Unlike mouse microglia, IBA-1-positive microglia in M. murinus and humans were larger and displayed bigger somas and thicker processes (Fig. 2E, F, arrowheads). GFAP immunostaining revealed a higher density of astrocytes in the white matter than in the gray matter of all 3 species (Fig. 2G-I). Within the gray matter of the mouse spinal cord, astrocytes displayed a stellate shape with multiple, nonoverlapping, branched processes (Fig. S2B, D, arrow), whereas in the white matter they were in close proximity to one another and exclusively presented an elongated morphology with numerous overlapping processes (Fig. 2J, arrowhead). Unlike in mice, GFAP-positive astrocytes in the white matter of M. murinus and humans displayed both stellate and elongated morphologies (Fig. 2K, L, arrows and arrowheads). In addition, GFAP-positive astrocytes were enlarged and displayed extended somas and thicker processes in primate spinal cords than those of rodents (Fig. 2J-L).

These results revealed that the spinal cord of M. murinus displays white-to-gray matter ratios and glial cell morphologies/distributions that closely resemble those of human spinal cords.
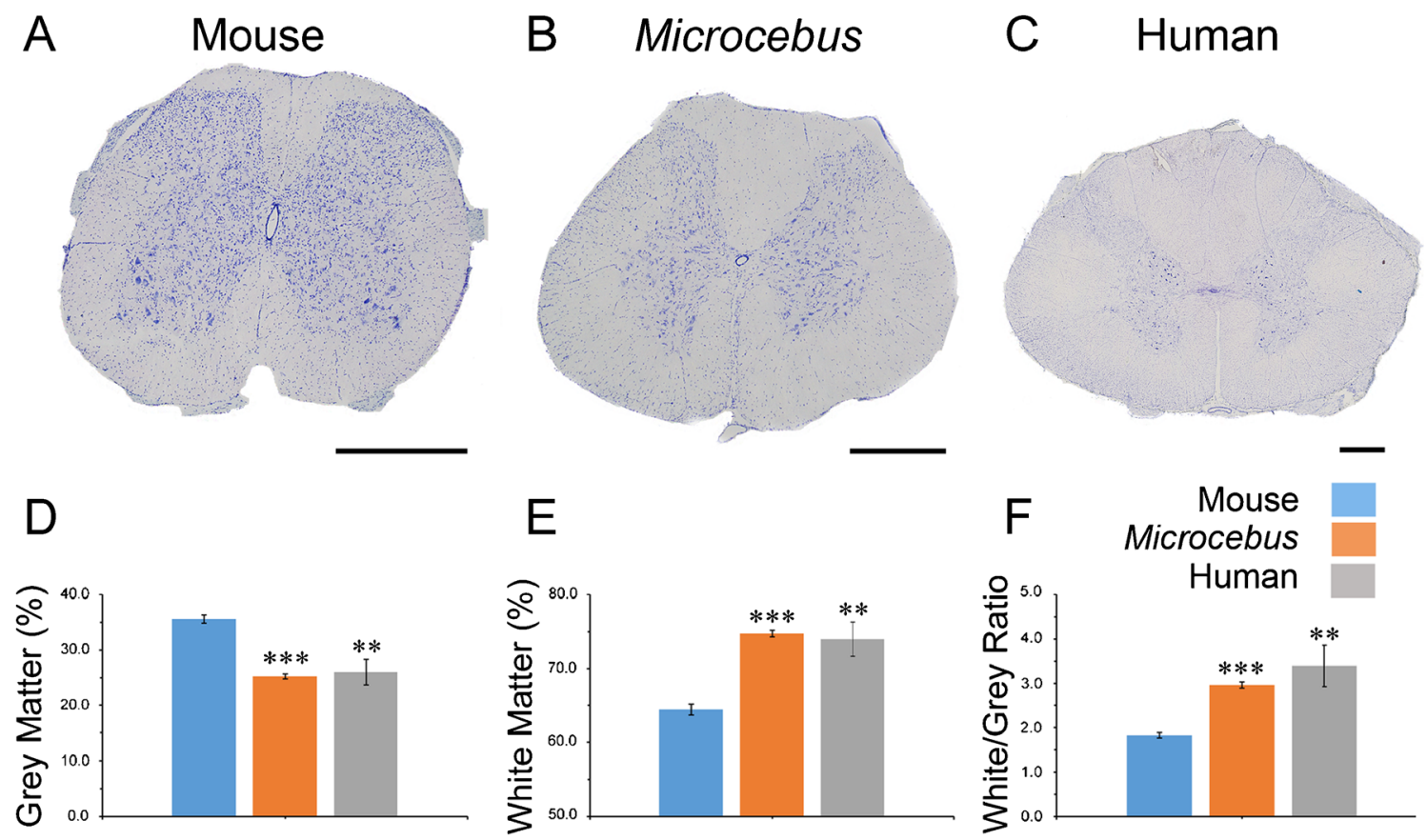

Fig. 1 Similar neuroanatomical features between Microcebus murinus and human spinal cords. Brightfield micrographs displaying differences in neuroanatomic features between (A) mouse, (B) M. murinus, and (C) human spinal cords at a low thoracic level using toluidine blue staining. Scale bar $=1 \mathrm{~mm}$. (D-F) Note that unlike mouse spinal cord, $M$. murinus and human spinal cords displayed a higher white-to-gray matter ratio. $* * p$ $<0.01$ and $* * * p<0.001$ (unpaired $t$-test) vs the value in mice. Eight mice, 3 noninjured M. murinus, and 15 humans. In all species, we analyzed a spinal cord segment of at least $300 \mu \mathrm{m}$ thickness 
Fig. 2 Similar glial cell distribution/morphology between Microcebus murinus and human spinal cords. Brightfield micrographs displaying glial cell distribution/morphology in (A, D, G, J) mouse, (B, E, H, K)

M. murinus, and (C, F, I, L) human spinal cords using (A-F) ionized calcium-binding adapter molecule 1 (IBA-1) and (G-L) glial fibrillary acidic protein (GFAP) immunohistochemistry. Note that in the mouse spinal cord microglia displayed typical ramified morphology having short/thin processes (arrow in D), whereas microglia were larger in size with enlarged soma and thicker processes in M. murinus and human spinal cords (A-F, arrowheads in $\mathrm{E}$ and $\mathrm{F}$ ). Likewise, astrocytes within the white matter of mouse spinal cord predominantly displayed elongated morphology with numerous overlapping processes (J, arrowhead), whereas in M. murinus and human astrocytes had both stellate and elongated morphologies ( $\mathrm{K}$ and $\mathrm{L}$, arrows and arrowheads). Scale bar (A-C, $\mathrm{G}-\mathrm{I})=1 \mathrm{~mm}$; (D-F, J-L) $=25$ $\mu \mathrm{m}$. Eight mice, 3 noninjured M. murinus, and 15 humans
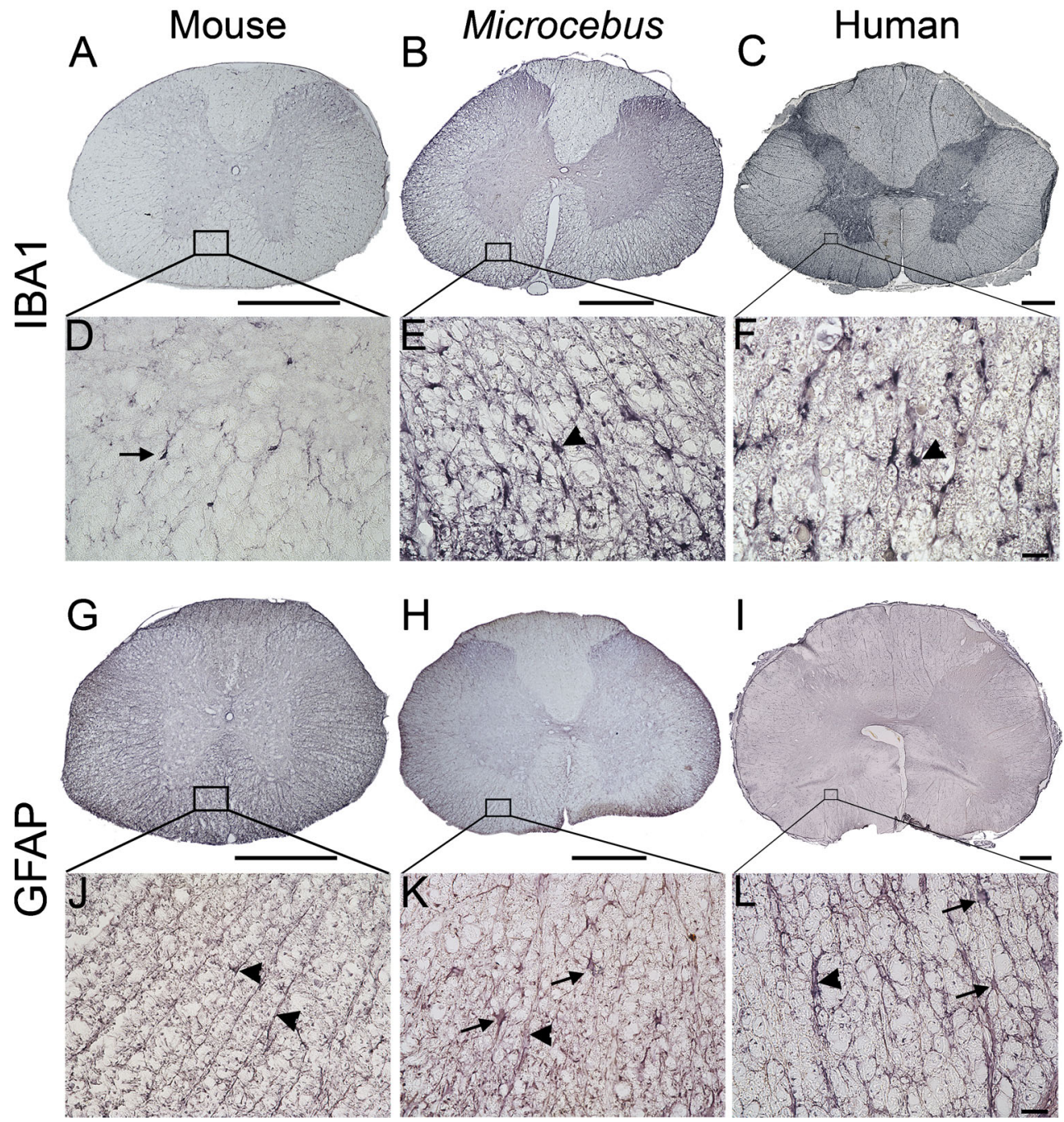

\section{Characterization of Motor Effects After Lateral Spinal Cord Hemisection in Microcebus murinus}

In the first series of experiments using 2 M. murinus, we standardized the surgical protocol, behavioral assessments, and in vivo ${ }^{1} \mathrm{H}$-MRI follow-up. In the second series, 3 additional animals were evaluated using behavioral tests and in vivo ${ }^{1} \mathrm{H}$-MRI evaluations at $48 \mathrm{~h}$, and 1,4 , and 12 weeks after SCI. Spinal cord hemisection was done at lumbar level 1 (Fig. S1A-C, arrows) to obtain a hindlimb monoplegia ipsilateral to the lesion while preserving full respiratory and bladder functions. All animals survived surgery and repetitive anesthesia ( ${ }^{1} \mathrm{H}-\mathrm{MRI}$ acquisition). One animal from the first series developed severe self-biting directed toward the paralyzed limb at 2 months after injury. The animal was treated with enrofloxacine $(5 \mathrm{mg} / \mathrm{kg} /$ day, 1 week; Bayer) associated with daily wound cleansing [Vetadine (Bayer) and occlusive bandages]. None of the animals required manual facilitation of bladder voiding. Body weight was recorded at 24 and $72 \mathrm{~h}$ postlesion followed by weekly assessments for 3 months postinjury (Fig. 3A). All animals were fasted 12 hours prior to each anesthesia required for surgery and for in vivo ${ }^{1} \mathrm{H}-\mathrm{MRI}$ acquisition. This resulted in 6\% to $9 \%$ weight loss (arrows in Fig. 3A). All animals quickly returned to their initial body weights. One M. murinus (number 1) had gained almost $10 \%$ of its body weight after the last in vivo ${ }^{1} \mathrm{H}-\mathrm{MRI}$ acquisition.

In the first series, we tested the feasibility of 3 different tests for motor function evaluation, namely the open-field, grip test, and CatWalk analyses. The open-field test revealed that the motor score of the hindlimb ipsilateral to the lesion dropped from a score of 6 (normal walking) to 0 to 1 (no or barely perceptible movements), which lasted up to 6 days following surgery (Fig. 3B). Between 6 and 14 days postlesion, the overall scoring showed gradual functional recovery followed by stabilization at around $80 \%$ of their initial scoring (mean score of 5; Fig. 3B). The contralateral limb showed a transient impairment up to day 30 postinjury. From day 33 after SCI, 2 M. murinus returned to normal and 1 remained at a score of 5.5 (very mild deficit; Fig. 3B). Statistical analysis showed 
Fig. 3 Characterization of functional outcomes after lateral spinal cord hemisection in Microcebus murinus. Weight curve of M. murinus over the 3 months following spinal cord hemisection. (A) Each curve represents one animal. (B) Line graph showing changes in motor hindlimb scores located ipsilateral and contralateral to the lesion in M. murinus. Values represent average of the group $(n=3)$. (C) Inclined barrier used to assess grip scores in M. murinus after spinal cord injury (SCI) and zoomed in on toes. (D) Line graph showing changes in grip scores between hindlimb toes located on the injured and uninjured sides of the spinal cord in M. murinus after SCI $[* * p<0.01, * * * p<0.001,2-$ way analysis of variance

(ANOVA)]. Walking patterns of M. murinus (E) before lesion, $(\mathrm{F})$ at $1,(\mathrm{G}) 14,(\mathrm{H})$ and 90 days after lateral spinal cord hemisection.

(E) Front paws are represented by glossy colors and the hindpaws are represented in matt. Line graphs displaying changes in (I) the base of support, $(\mathrm{J})$ regularity index, $(\mathrm{K})$ swing, and $(\mathrm{L})$ maximal area in M. murinus for 3 months after SCI $(* * p<0.01$, $* * * p<0.001,1$ way ANOVA).

Time after SCI in days.

M. murinus: $n=3$ (M1, M2, M3)
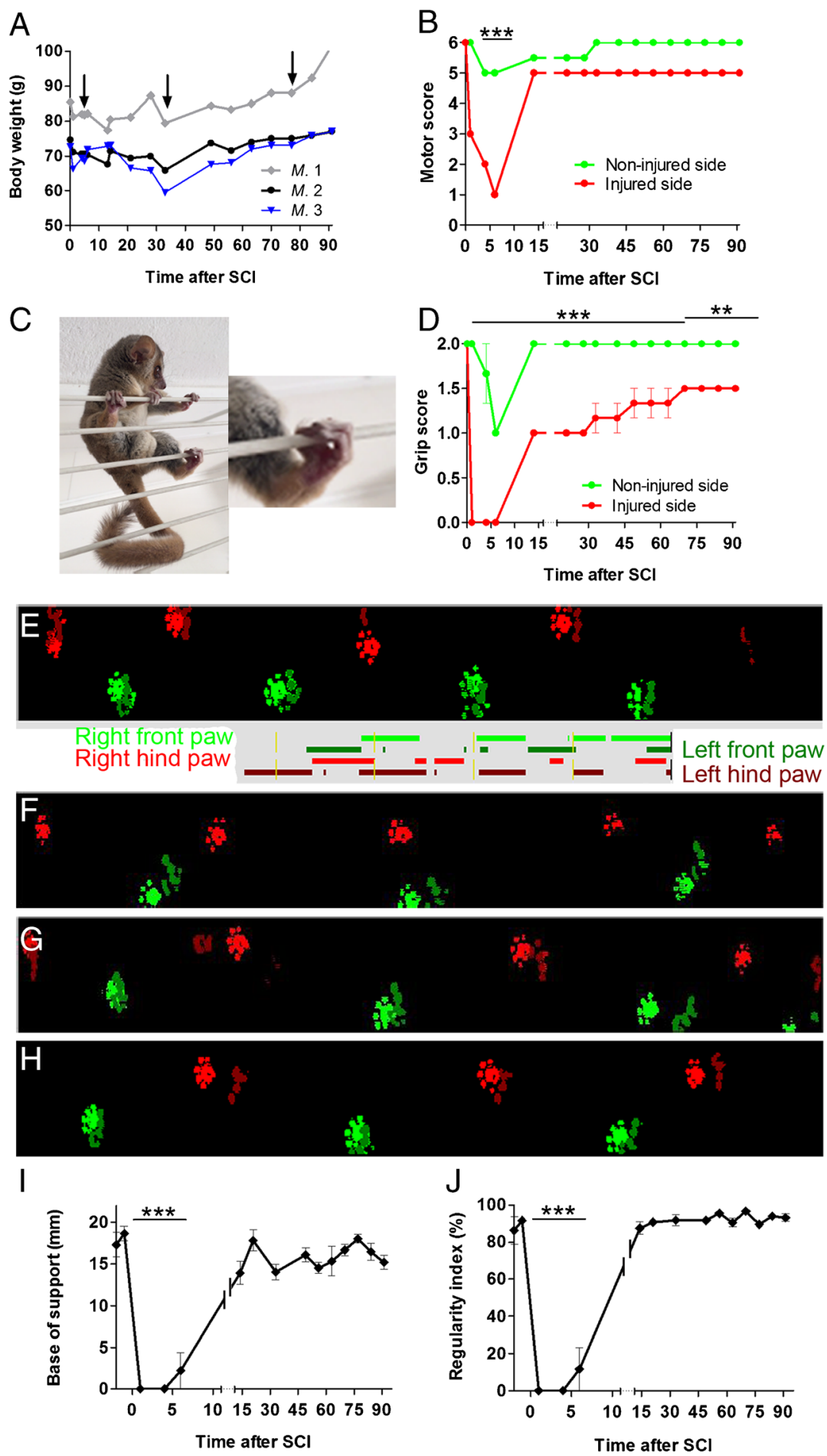

$\mathrm{K}$
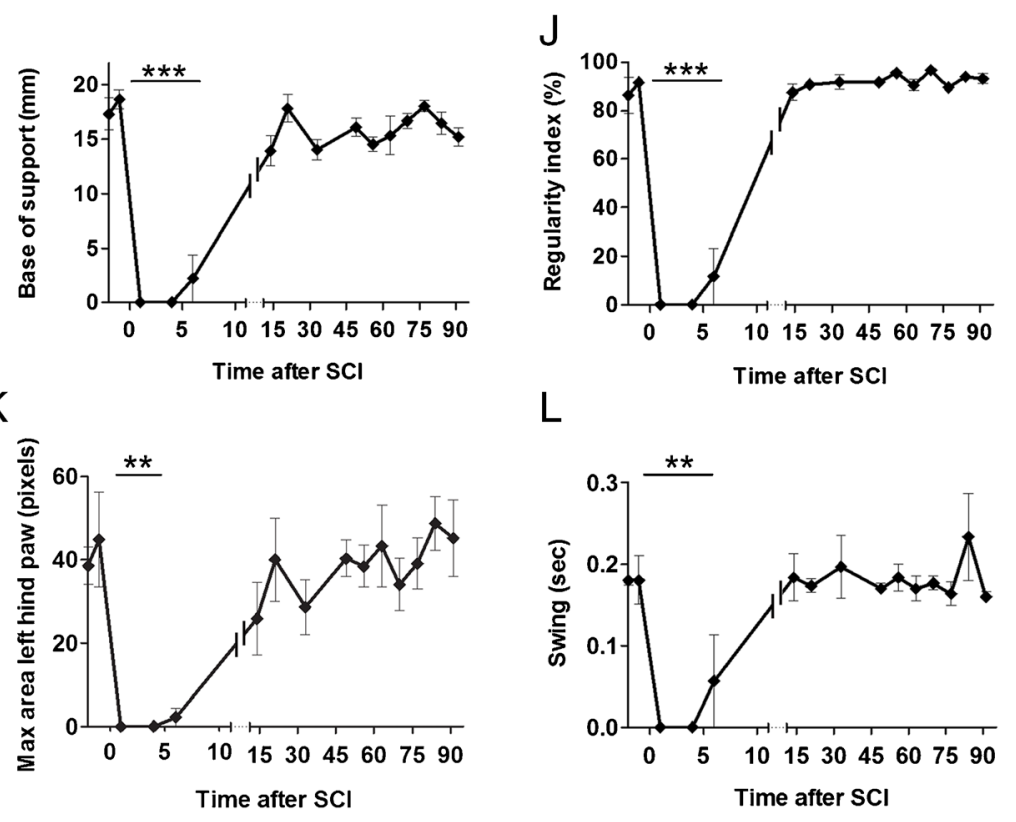

L

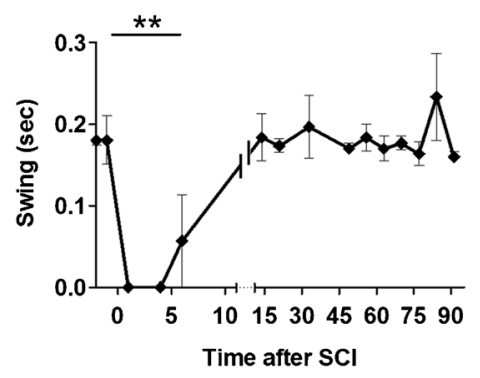


significant difference between the 2 hindlimbs up to 6 days postinjury ( $p<0.001$; Fig. 3B).

To evaluate fine motor function, we used the grip test (Fig. 3C). The grip score of the ipsilateral hindlimb toes dropped from normal grip (score of 2) to no grip (score of 0 ) in the 6 days following surgery; then, a gradual, but partial, recovery was observed that plateaued for all animals at $75 \%$ of their initial scoring from 70 to 90 days postinjury (time of the study; Fig. 3D). The contralateral limb showed a transient impairment in the 6-day postinjury period that subsequently returned to normal by day 14 in all animals (Fig. 3D). Statistical analysis revealed a significant difference between the 2 hindlimbs up to 90 days postinjury (Fig. 3D).

To better quantify walking deficits, we also performed automated gait analysis using CatWalk recordings (Fig. 3E-L). Out of all parameters that were assessed, 2 dynamic gait parameters were the most accurate for evaluating motor function following SCI: 1) regularity index, which measures interpaw coordination; and 2) duration of the swing phase, which is the time duration without contact between a given paw and the glass plate in a step cycle. We also analyzed 2 static parameters: 1) the base of support, defined as the width between the hindpaws; and 2) the maximal area of the hind paw ipsilateral to the lesion, which corresponds to the print area at maximum contact. Between 1 and 4 days after SCI, both dynamic and static parameters decreased to 0 for all animals and then gradually increased within 14 days following lateral hemisection (Fig. 3I-L). Statistical analysis showed no significant difference in swing after 6 days postinjury, whereas the base of support, the regularity index, and the maximal area remained significantly low until 14 days postlesion compared with before the injury (Fig. 3I-L).

These results showed that all current tests can accurately evaluate alterations in motor function of the ipsilateral hindlimb up to 2 weeks following injury. However, only the grip test clearly discriminates between hindlimbs on the injured and noninjured sides of the spinal cord throughout the experimental period (3 months).

Altogether, we have developed a reproducible model of lateral spinal cord hemisection in M. murinus and designed a sequential combination of behavioral tests that allow accurate monitoring of motor function after SCI.

\section{Longitudinal in vivo ${ }^{1} \mathrm{H}-\mathrm{MRI}$ Follow-Up}

We also analyzed lesion evolution over 3 months postinjury through longitudinal acquisition of axial in vivo ${ }^{1} \mathrm{H}$-MRI. Images were obtained for each animal at $48 \mathrm{~h}, 1$ and 4 weeks, and 3 months after the lesion (Fig. 4). We identified tissue modifications at the lesion site over time (Fig. 4). Specifically, at $48 \mathrm{~h}$ after injury, a hyposignal was identified in the perihematomal region on the hemisected side of the spinal cord (Fig. 4C, outlined in pink in Fig. 4D), contrasting with the normal hypersignal of the gray matter on the opposite side (Fig. 4C, outlined in green in Fig. 4D). This hyposignal decreased progressively but remained visible at 1 (Fig. 4E) and 4 weeks postinjury (Fig. 4F). At 12 weeks postinjury, the lesion site appeared as a hypersignal (Fig. 4G). Both signal intensities (hypo- and hypersignals) were associated with disorganization of the spinal cord tissue (Fig. 4C-G). To quantify lesion extension over the 3 months postinjury, we analyzed a 1.6- $\mathrm{cm}$ spinal cord segment centered on the lesion epicenter. Lesion segmentation was carried out through surface area analysis by outlining the spared and the injured white and gray matter (Fig. 4B, D). The percentage of surface area occupied by the lesion at the epicenter was $46.4 \pm 2.6 \%$; $47.2 \pm 3.2 \%$; $42 \pm 3.5 \%$, and $32.8 \pm 6.2 \%$ at $48 \mathrm{~h}, 1$ and 4 weeks, and 3 months postinjury, respectively (Fig. 4I). Lesion extension was more pronounced caudal to the lesion $(2.3 \pm 0.3 \mathrm{~mm})$ than rostral $(1.4 \pm 0.1 \mathrm{~mm})$ at 3 months postinjury (Fig. $4 \mathrm{I})$. Quantitative assessments showed no significant difference in lesion evolution over time (Fig. 4I). However, areas under the curve (AUC) showed a peak at 1 week (AUC of 153) versus all other time points (AUCs of 133, 127, and 106 for $48 \mathrm{~h}, 4$ weeks, and 3 months, respectively); the lowest total area was observed at 3 months following SCI (Fig. 4I). Lesion volume over time, though not statistically significant, followed a similar evolution pattern with a peak at 1 week and a lower value at 3 months following SCI (Fig. 4J).

These results show that in vivo ${ }^{1} \mathrm{H}-\mathrm{MRI}$ longitudinal follow-up allows quantitative assessment of lesion evolution over time in M. murinus after a lateral hemisection of the spinal cord.

\section{Ex vivo ${ }^{1} \mathrm{H}$-MRI Assessment of the Lesion}

We next performed ex vivo ${ }^{1} \mathrm{H}-\mathrm{MRI}$ analysis, as previously described [30, 31, 34], because it allows an amplification of the signal-to-noise ratio versus in vivo ${ }^{1} \mathrm{H}$-MRI and thus permits better discrimination between spared and injured spinal cord tissue postinjury. At 3 months following SCI, whole spinal cords were imaged and underwent segmentation (Fig. 5). At the lesion epicenter, the hemisected side appeared with a hyperintense signal that covered half of the spinal cord (Fig. 5B), whereas rostral (Fig. 5A) and caudal to the lesion site (Fig. 5C) the gray and the white matter were complete and clearly identifiable. Moreover, caudal to the lesion, a limited but significant atrophy was apparent in the dorsal horn and mediodorsal funiculus on the injured side (Fig. 5C). The lesion extended evenly a distance of 1.6 $\pm 0.9 \mathrm{~mm}$ on both sides of the epicenter along the rostrocaudal axis, and the lesion percentage at the epicenter was $35.9 \pm 6.7 \%$ (Fig. 5F).

Thus, lesion extension quantified using in and ex vivo ${ }^{1} \mathrm{H}$-MRI were in similar ranges, i.e., the extension 
Fig. 4 Longitudinal in vivo ${ }^{1} \mathrm{H}-$ magnetic resonance imaging (MRI) assessment of spinal cord injury in M. murinus. In vivo axial images within the lesion epicenter taken at (c, d) $48 \mathrm{~h}$, (E) 1 week, (F) 4 weeks, and (G) 3 months after spinal cord hemisection relative to $(\mathrm{A}, \mathrm{B})$ rostral and $(\mathrm{H})$ caudal to the lesion site. (B, D) Drawings of the spared gray matter (green), the entire spinal cord (blue), and (D) the injured tissue (pink). Longitudinal quantitative assessments of (I) lesion extension and $(\mathrm{J})$ volume extension at different stages after lateral spinal cord hemisection in M. murinus $(n=3)$
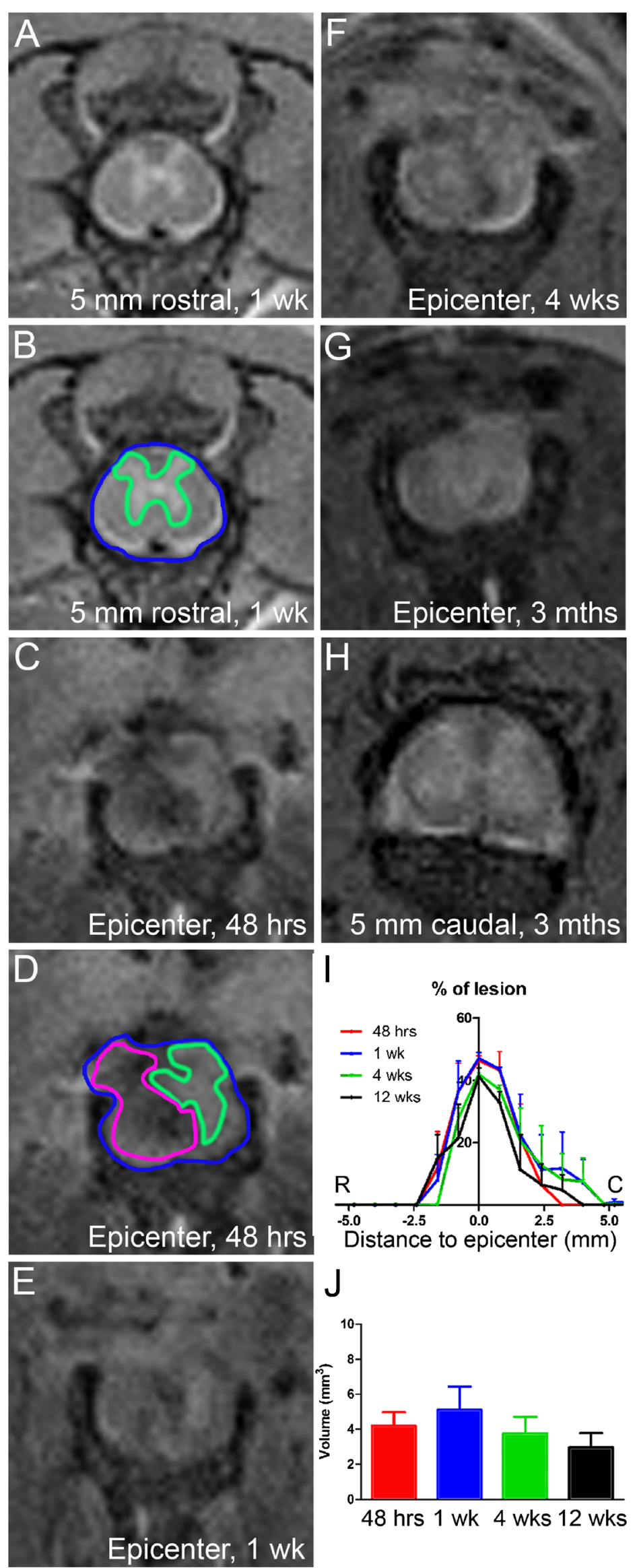
Fig. 5 Ex vivo ${ }^{1} \mathrm{H}$-magnetic resonance imaging (MRI) assessments and correlation between ${ }^{1} \mathrm{H}-\mathrm{MRI}$ signals and microglia/macrophage reactivity within the dorsal funiculus. ${ }^{1} \mathrm{H}$ MRI image of ex vivo spinal cord from Microcebus murinus (A) rostral, (B) within, and (C) caudal to the lesion site. Note the excellent anatomic resolution of the spinal cord due to the high signal-to-noise ratio and high isotropic resolution achieved after incubation first in the gadolinium then in Fluorinert combined with longer acquisition time. $(\mathrm{F})$ Line graph showing quantitative assessment of the lesion extension at 3 months after lateral spinal cord hemisection in M. murinus. (E, G) Immunohistochemical staining using ionized calcium-binding adapter molecule 1 (IBA-1) antibody of the spinal cords from M. murinus (E) rostral and (G) caudal to the lesion site relative to their corresponding ex vivo ${ }^{1} \mathrm{H}$ MRI (A and C, respectively). In the dorsal funiculus, the hyperintense signal in ex vivo ${ }^{1} \mathrm{H}$ MRI coincides with increased microglia/macrophage reactivity rostral (red arrows in A and E) to the lesion site. (D) Line graphs displaying quantitative analysis of signals from the gray matter (blue), the entire dorsal funiculus (red), and the dorsal funiculus ipsilateral to the lesion that display hyper- (rostral to the lesion) or hypo- (caudal to the lesion) signals (green). Quantifications were done at different distances to the lesion site using either (D) ex vivo or $(\mathrm{H})$ in vivo ${ }^{1} \mathrm{H}-\mathrm{MRI}$ at 12 weeks postinjury. Scale bar (A-E, G): 1 $\mathrm{mm}$. Straight lines $=$ double linear regression (rostral and caudal to the lesion site represented as 0 on the axis). M. murinus: $n=5$ for ex vivo ${ }^{1} \mathrm{H}-\mathrm{MRI}$ quantification and $n=3$ for in vivo ${ }^{1} \mathrm{H}-\mathrm{MRI}$
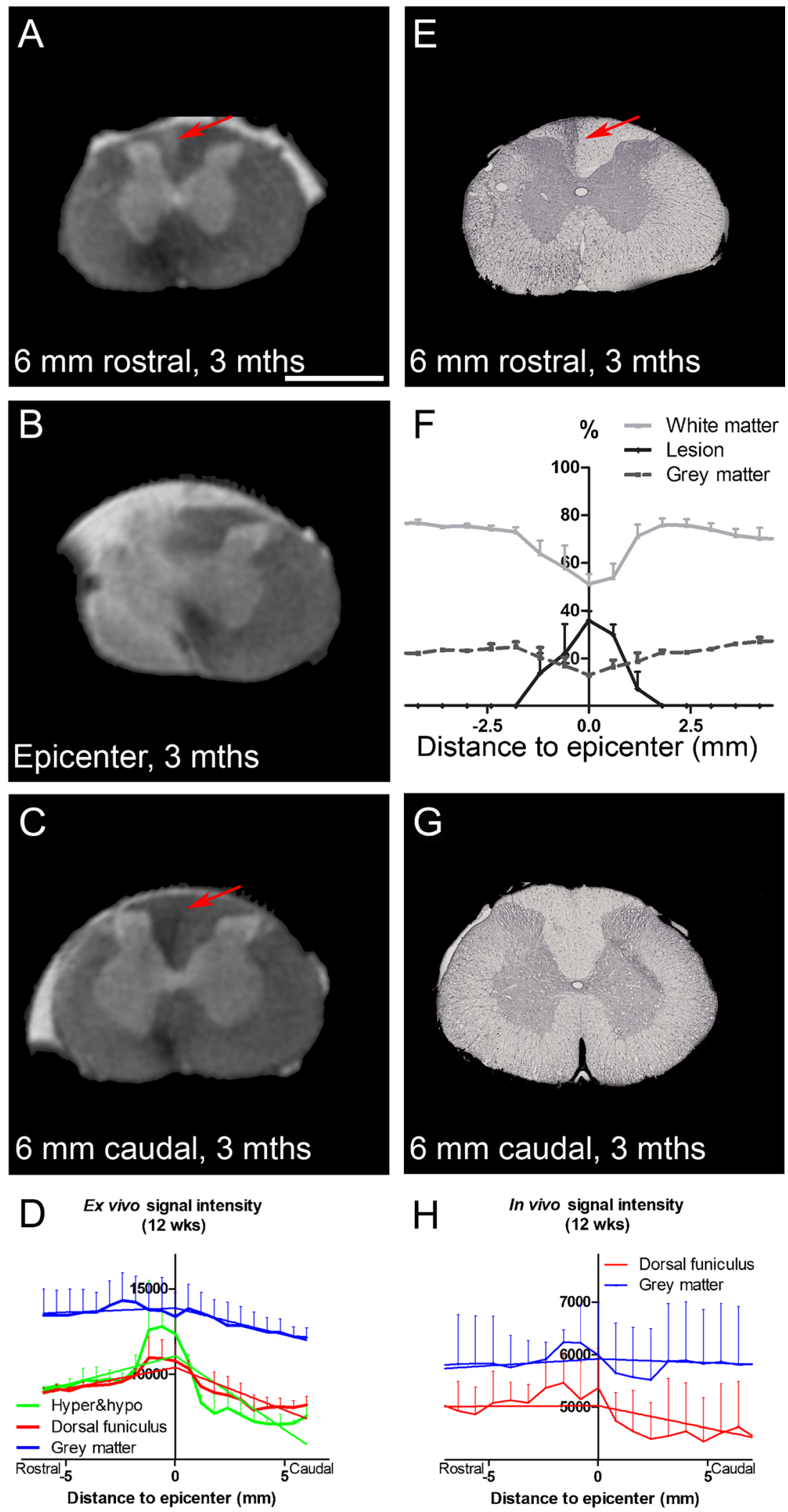

measured in vivo at 3 months postlesion was $3.4 \pm 2 \mathrm{~mm}$ and that using ex vivo ${ }^{1} \mathrm{H}-\mathrm{MRI}$ was $1.6 \pm 0.9 \mathrm{~mm}$. Similarly, the percentage of damaged tissue was quantified at $32.8 \%$ in vivo and $35.9 \%$ ex vivo. 


\section{Comparison Between in vivo ${ }^{\mathbf{H}} \mathrm{H}-\mathrm{MRI}$, High-Resolution ex vivo ${ }^{1} \mathrm{H}-\mathrm{MRI}$, and Histological Observations}

We then examined putative correlation 3 months after SCI between the 3 methods being analyzed: in vivo ${ }^{1} \mathrm{H}$-MRI at the end of the experiment, ex vivo ${ }^{1} \mathrm{H}-\mathrm{MRI}$, and histological data (Figs S3A-C, S4, S5). At the epicenter, the lesion area [1 single section corresponding to the epicenter (Fig. S3B), quantification (Fig. S3D, E)] showed no statistical difference between the 3 methods with approximately $40 \%$ of the spinal cord tissue being damaged (Fig. S3E). Quantification of lesion volume was also similar between ex vivo ${ }^{1} \mathrm{H}-\mathrm{MRI}$ and histology (AUCs of 65.4 and 53.09, respectively; Fig. S3F). However, lesion volume on the rostrocaudal axis was greater (AUC of 106.4) using in vivo ${ }^{1} \mathrm{H}-\mathrm{MRI}$ (Fig. S3E). Since lesion volume may be more representative of the actual tissue damage, we used the 3 different methods to compare the volume at 3 months after SCI (Fig. S3F). Even though lesion volume using in vivo ${ }^{1} \mathrm{H}-\mathrm{MRI}$ appeared greater than with histology, this difference did not reach statistical significance, most likely owing to the small number of animals (Fig. S3F).

Taken together, these results show that there is no statistically significant difference in the evaluation of damaged tissues using the 3 different methods.

\section{Glial Reactivity After Lateral Spinal Cord Hemisection in Microcebus murinus}

We next used GFAP and IBA-1 immunostaining to examine SCI-induced glial reactivity in M. murinus at 3 months following trauma (Figs 6,7). A pronounced increase in the expression of GFAP was observed at the lesion epicenter versus the regions rostral and caudal to the injury site (Fig. 6A-I). Astrocytes adjacent to the lesion site displayed elongated morphology with numerous overlapping processes close to one another (Fig. 6E, F). Quantitative analysis confirmed the increase in GFAP expression adjacent to the lesion site (Fig. 6J, K). However, we observed a significant increase in GFAP only in the gray matter rostral to the lesion site compared with caudal segment (Fig. 6J).

Similarly, we observed an increase in IBA-1 immunoreactivity adjacent to the lesion epicenter versus rostral and caudal segments (Fig. 7A-I). We also found pronounced microglia/macrophage reactivity within the dorsal funiculus rostral to the lesion site (Fig. 7A-C). Morphologically, the IBA-1-positive microglia/macrophages could be categorized into 2 groups: 1) ramified/resting cells displaying small cell bodies with randomly distributed thin-tomedium-sized processes (Fig. 7C, I, arrows); and 2) reactive/hypertrophied microglia/macrophages with enlarged cell bodies and short/thick processes (Fig. 7C, F, arrowheads). Reactive/hypertrophied microglia/ macrophages were predominant not only at the lesion site, but also in the dorsal funiculus ipsilateral and rostral to the lesion (Fig. 7A-F, arrowheads in C, F), whereas only ramified microglia were evident caudal to the lesion (Fig. 7G-I, arrows in I). Quantitative analysis within the gray matter and the dorsal funiculus confirmed a significant increase in IBA-1 expression rostral to the lesion compared with caudal regions (Fig. 7J, L). IBA-1 expression was also increased within the white matter adjacent to the lesion; however, there were no differences between the rostral and caudal segments (Fig. 7K).

These data reveal that at 3 months following SCI in M. murinus 1) glial reactivity increases adjacent to the lesion site compared with more distal segments; 2) there is a significant increase in astrocyte and microglia/ macrophage reactivity within the gray matter rostral to the lesion but not caudal; and 3) the dorsal funiculus on the lesion side displays a pronounced increase in microglia/macrophage reactivity rostral to the lesion site versus the caudal segment.

\section{Correlation Between Increased Microglial Reactivity and ${ }^{1}$ H-MRI Signals}

Given the pronounced microglia/macrophage reactivity in the dorsal funiculus, we then carried out a more in-depth analysis of ${ }^{1} \mathrm{H}$-MRI signal intensities to determine the possibility of comparing ${ }^{1} \mathrm{H}$-MRI signals with microglia/macrophage immunoreactivity. Using ex vivo ${ }^{1} \mathrm{H}-\mathrm{MRI}$, we observed a hyperintense signal within the dorsal funiculus, only rostral to the lesion site (Fig. 5A, arrow; Fig. S5D, G, arrows), that coincided precisely with the area of increased microglia/ macrophage immunoreactivity (Fig. 5E, arrow). Conversely, a hyposignal was observed within the dorsal funiculus caudal to the lesion site (Fig. 5C, arrow; Fig. S5C, F, I, arrows) that coincided with an absence of microglia/macrophage reactivity (Fig. 7G-I). Quantification of the ${ }^{1} \mathrm{H}-\mathrm{MRI}$ signal in ex vivo showed a lower overall signal in the gray matter rostral to the lesion site versus caudal (Fig. 5D, blue curve). This difference in signal intensity was more evident when only the dorsal funiculus was analyzed (Fig. 5D, red curve) and even further increased when focusing on the portion of the dorsal funiculus ipsilateral to the lesion (Fig. 5D, green curve).

We then analyzed in vivo ${ }^{1} \mathrm{H}-\mathrm{MRI}$ signals within the gray matter and the dorsal funiculus using ${ }^{1} \mathrm{H}-\mathrm{MRI}$ axial images. Owing to high signal variability, it was not possible to identify differences within the dorsal funiculus rostral and caudal to the lesion at 1 and 4 weeks postinjury (Fig. S6A, B). However, a hypersignal was observed 3 months following SCI in the dorsal funiculus of the segment rostral to the lesion site versus the caudal segment (Fig. 5H, Fig. S6C). 

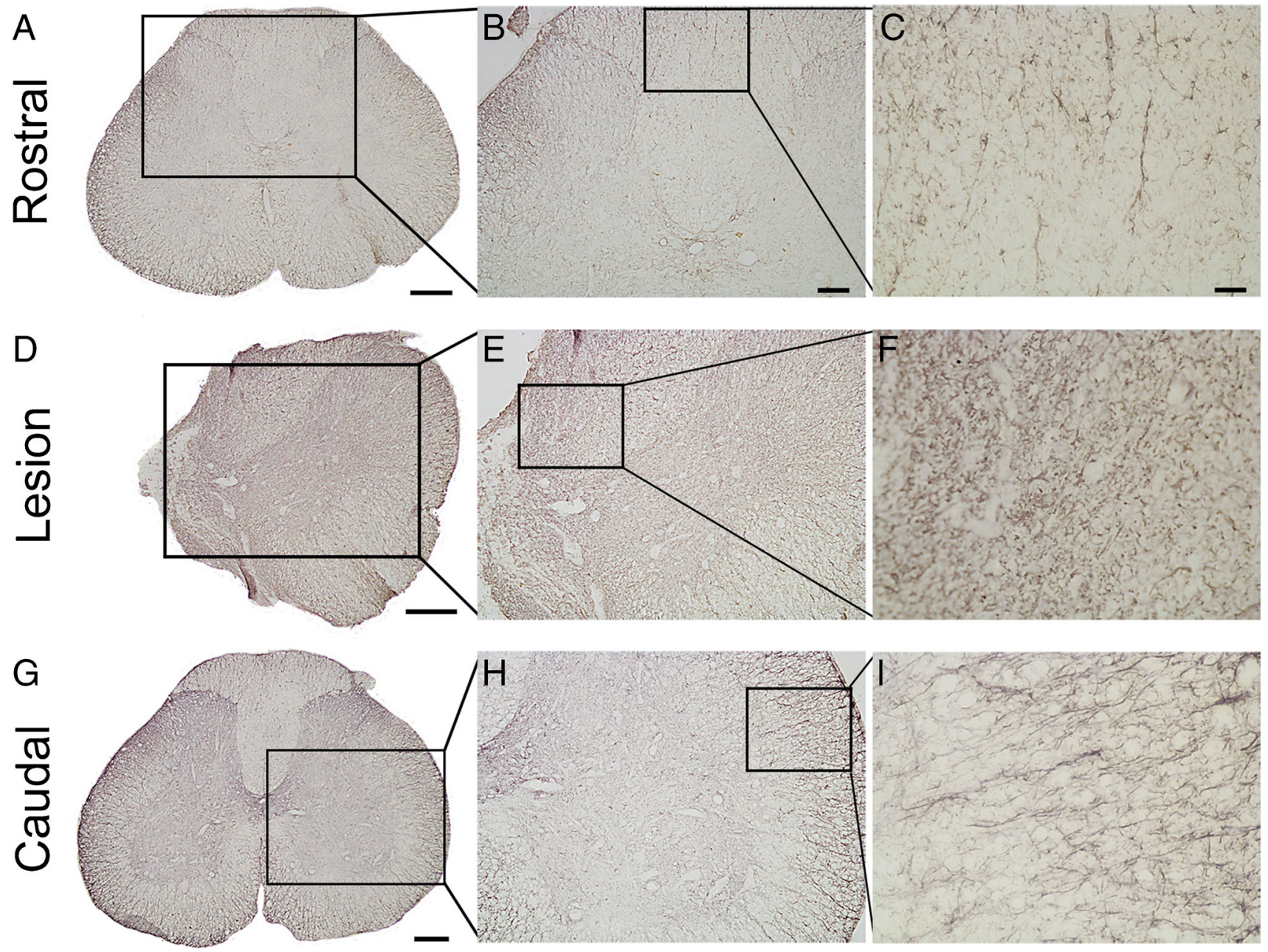

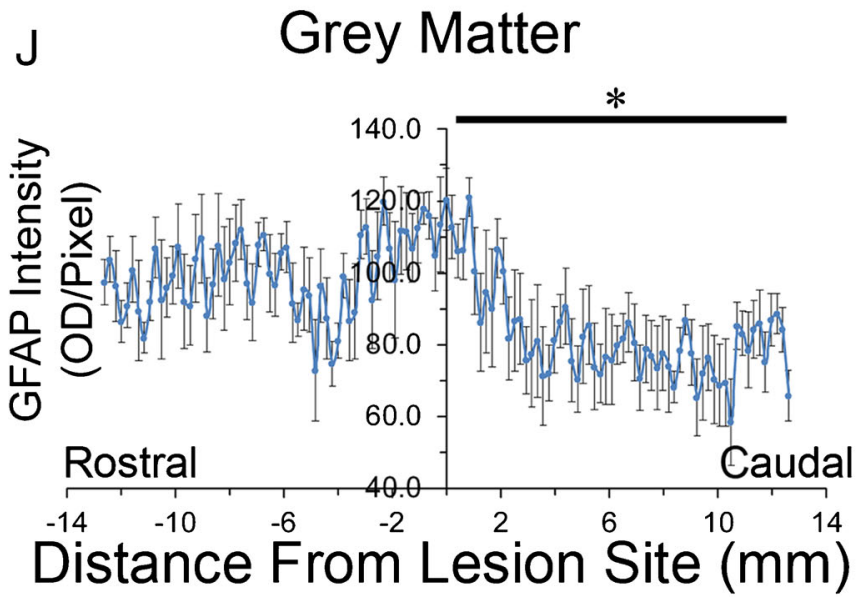

Fig. 6 Astrocytic reactivity 3 months after spinal cord injury (SCI) in Microcebus murinus. Brightfield micrographs displaying glial fibrillary acidic protein-positive astrocytes (A-C) rostral, (D-F) within, and (G-I) caudal to the lesion site at 3 months after SCI in M. murinus. Astrocytes displayed typical stellate and elongated morphology with multiple nonoverlapping branched processes both $(\mathrm{B}, \mathrm{C})$ rostral and $(\mathrm{H}, \mathrm{I})$ caudal to the lesion site, whereas $(\mathrm{E}, \mathrm{F})$ reactive astrocytes within the lesion site showed irregular morphology with numerous overlapping
K White Matter

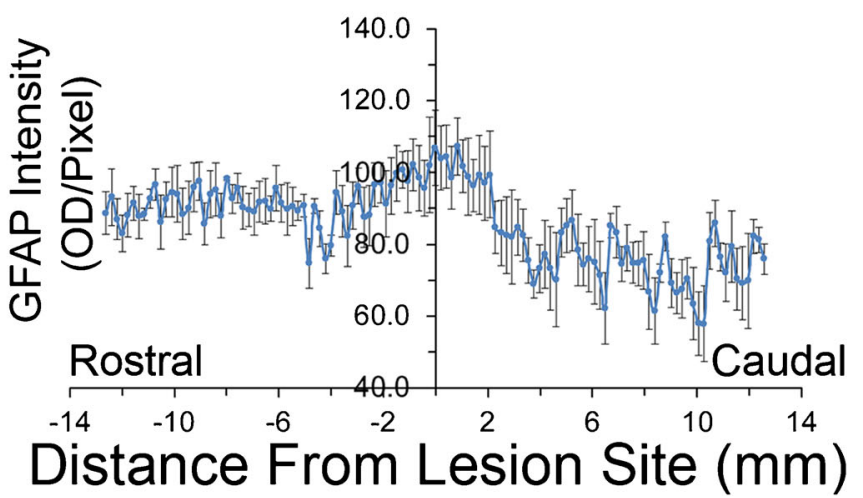

processes. Line graphs displaying quantitative assessment of astroglial reactivity within the $(\mathrm{J})$ gray and the $(\mathrm{K})$ white matters of the spinal cord at different distances to the lesion epicenter. Note the increase in astroglial reactivity after lesion within the gray matter rostral to the lesion site relative to caudal regions of the spinal cord. $* p<0.05$ paired $t$-test $v s$ similar distance rostral to the lesion site. Scale bar $(\mathrm{A}, \mathrm{D}, \mathrm{G})=250$ $\mu \mathrm{m} ;(\mathrm{B}, \mathrm{E}, \mathrm{H})=100 \mu \mathrm{m} ;(\mathrm{C}, \mathrm{F}, \mathrm{I})=25 \mu \mathrm{m}$. M. murinus: $n=5$ 

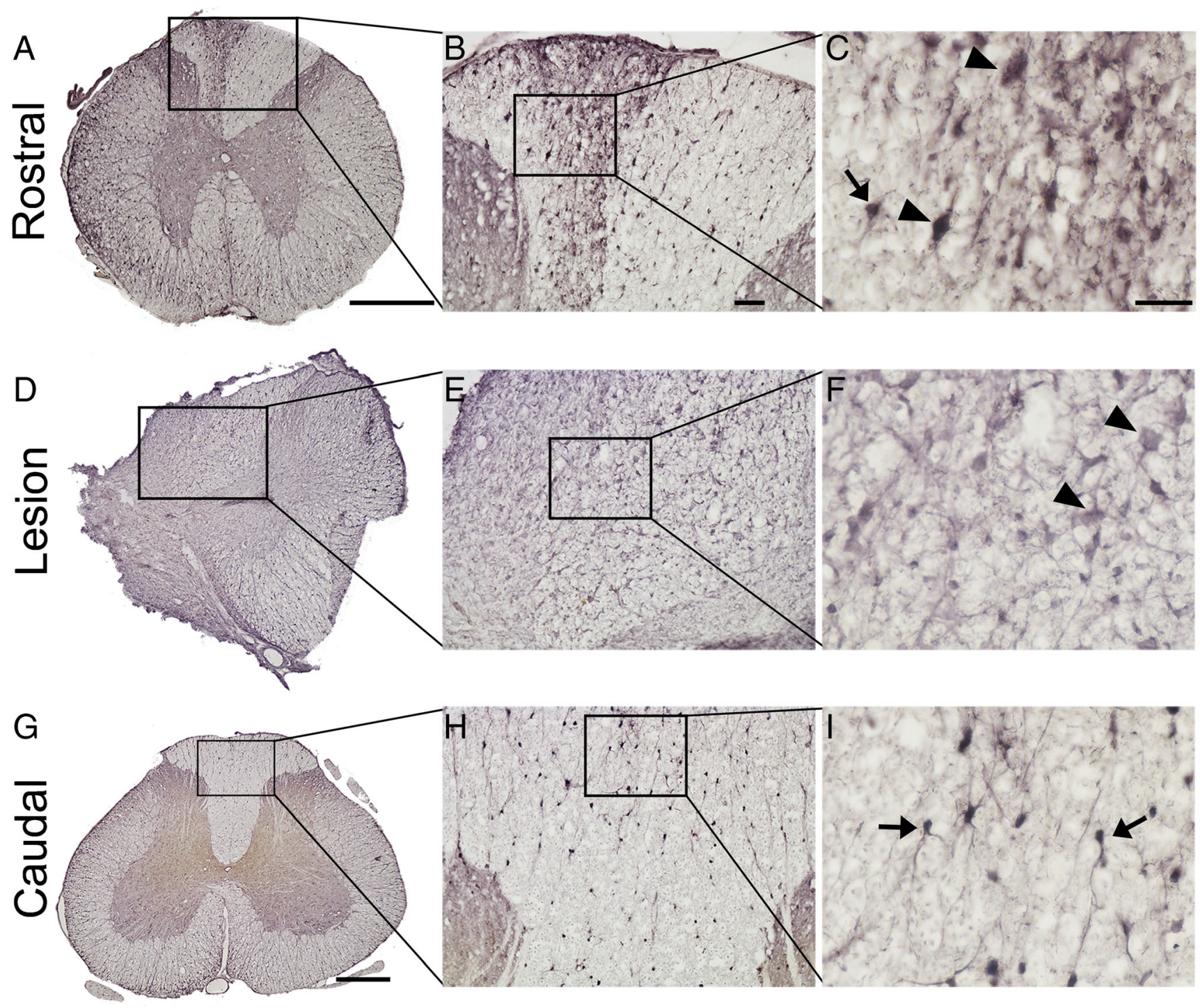

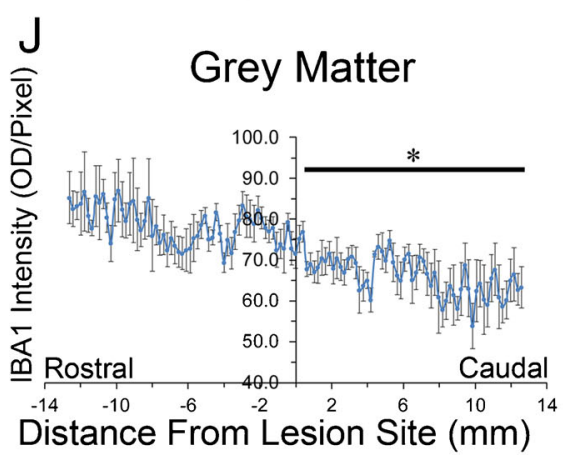

Fig. 7 Microglia/macrophage reactivity 3 months after spinal cord injury (SCI) in Microcebus murinus. Brightfield micrographs displaying ionized calcium-binding adapter molecule 1 (IBA-1)-positive microglia/ macrophage $(\mathrm{A}-\mathrm{C})$ rostral, $(\mathrm{D}-\mathrm{F})$ within, and $(\mathrm{G}-\mathrm{I})$ caudal to the lesion epicenter at 3 months after lateral spinal cord hemisection in M. murinus. Note that microglia displayed typical ramified morphology with short and thin processes both rostral and caudal to the lesion site (B and $\mathrm{C}$, arrow in $\mathrm{C}$; $\mathrm{H}$ and I, arrow in I), whereas reactive/hypertrophied microglia/macrophage with enlarged cell bodies and thick processes were localized adjacent to the lesion epicenter ( $\mathrm{E}$ and $\mathrm{F}$, arrowheads in F). Also, note that rostral to the

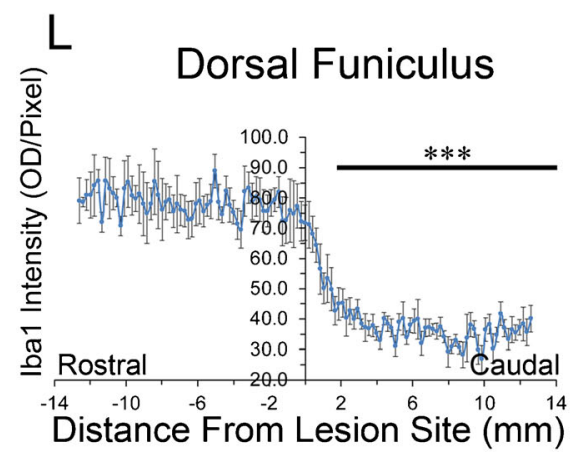

lesion site, the increase in microglia/macrophage reactivity persists within the dorsal funiculus up to $14 \mathrm{~mm}$ distal from the lesion site (A-C and $\mathrm{L}$, arrowheads in C). Line graphs displaying quantitative assessment of microglia/macrophage reactivity within $(\mathrm{J})$ the gray matter, $(\mathrm{K})$ the white matter, and (L) the dorsal funiculus of the spinal cords at different distances to the lesion epicenter. Note the increase in microglia/macrophage reactivity within the $(\mathrm{C}, \mathrm{F})$ gray matter and the dorsal funiculus rostral to the lesion site $v s$ (I) caudal regions of the spinal cord after lesion. * $p<0.05$, *** $p<0.001$ paired $t$-test $v s$ similar distance rostral to the lesion site. Scale bar $(\mathrm{A}, \mathrm{D}, \mathrm{G})=$ $250 \mu \mathrm{m}$; (B, E, H) $=100 \mu \mathrm{m}$; (C, F, I) $=25 \mu \mathrm{m}$. M. murinus: $n=5$ 
These results show that a pronounced increase in microglia/macrophage reactivity coincides with a hypersignal within the dorsal funiculus rostral to the lesion using both ex vivo and in vivo ${ }^{1} \mathrm{H}-\mathrm{MRI}$ analyses at 3 months after SCI.

\section{Alterations in NMJ Density After SCl in Microcebus murinus}

Finally, we examined the gastrocnemius, soleus, and plantaris muscles and quantified the number of NMJ on both hindlimbs (Fig. 8). We did not notice any differences in muscle surface area within the gastrocnemius-soleus-plantaris complex between the injured and the noninjured sides (Fig. 8A-E). However, quantification of NMJ density $\left(\mathrm{NMJ} / \mathrm{mm}^{2}\right)$ showed a strong decrease on the injured side (Fig. 8F).

\section{Discussion}

First, comparisons of the spinal cords of $M$. murinus with those of mice and humans highlighted stronger similarities in the general architecture, as well as glial components among primates than between mice and humans. Second, we have established a new model of SCI in a small NHP through a longitudinal behavioral evaluation and ${ }^{1} \mathrm{H}$-MRI follow-up that allowed the assessment of both motor impairments and in vivo lesion evolution. Third, 2 methods of ex vivo analysis, i.e., ex vivo ${ }^{1} \mathrm{H}-\mathrm{MRI}$ and immunohistochemical assessments, allowed the precise quantification of damaged tissue and glial reactivity. Microcebus murinus displayed significant microglia/macrophage and astrocyte reactivity within the gray matter rostral to the lesion epicenter. Moreover, the segment of the dorsal funiculus ipsilateral to the lesion side exhibited a
Fig. 8 Decreased neuromuscular junction density within the gastrocnemius-soleus-plantaris complex 3 months after spinal cord injury in Microcebus murinus. Brightfield micrographs showing gastrocnemius-soleusplantaris complex of the hindlimbs located on the (A, C) uninjured and $(\mathrm{B}, \mathrm{D})$ injured side of the spinal cord from $M$. murinus 3 months after lateral hemisection. Neuromuscular junctions were revealed using the Karnovsky and Roots enzymatic method. Bar graphs displaying quantitative assessment of (E) the surface area and (F) the density of neuromuscular junctions between muscles located on the uninjured and injured side of the spinal cord. $* * * p<0.001$ paired $t$-test $v S$ uninjured side. Scale bar $(\mathrm{A}, \mathrm{B})=$ $1 \mathrm{~mm} ;(\mathrm{C}, \mathrm{D})=50 \mu \mathrm{m} . M$. murinus: $n=5$.
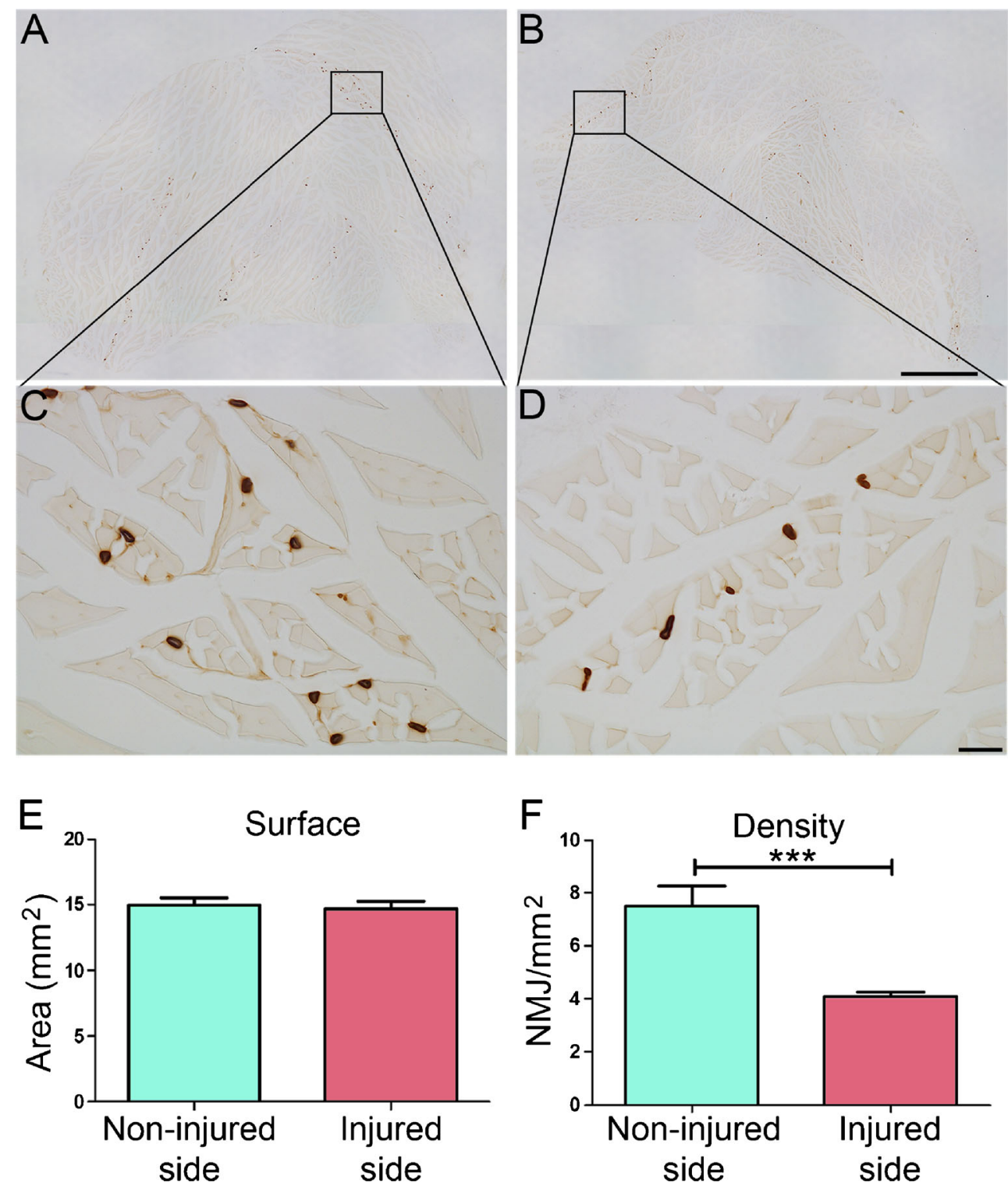
pronounced increase in microglia/macrophage reactivity rostral to the lesion site that precisely coincided with a hypersignal identified in both in and ex vivo ${ }^{1} \mathrm{H}-\mathrm{MRI}$ analyses. Finally, the peripheral consequences of SCI were characterized by a strong decrease in NMJ density within the gastrocnemius muscle located on the injured side of the spinal cord.

Anatomic knowledge of the spinal cord in M. murinus is extremely limited; only 1 study reported that early development of the neural tube is similar between M. murinus and humans [37]. To better document the anatomic organization of the rachis and the spinal cord of M. murinus we initially carried out a mini-atlas using computed tomography scan, ex vivo diffusion MRI, and histological staining. In the adult spinal cord, we found that the white-to-gray matter ratio was not only similar between M. murinus and humans, but that both were also significantly higher than in rodents. This is consistent with a previous study in the neocortex showing that white matter constitutes around $10 \%, 50 \%$, and $55 \%$ of the total volume in mice, lemurs, and humans, respectively [38]. The white matter contains non-neuronal cell bodies and axons; a higher percentage of white matter in primates than rodents may thus result from an increase in neuronal extension [39]. It is important to note that white-to-gray matter ratio is rather an abridge assessment to demonstrate similarities between species. Detailed neuroanatomical analyses, including morphometric analysis of neurons between M. murinus and human, are necessary to confirm these findings. The higher ratio of white-to-gray matter may also result from several other parameters including the number, density, and size of glial cells, the degree of axonal myelination, and differences in axonal caliber. In line with these possibilities, we observed that microglia and astrocytes are larger and display thicker processes in M. murinus and humans than those of mouse spinal cords. This is also in agreement with a study in the neocortex reporting that human protoplasmic and fibrous astrocytes have a larger diameter than their rodent counterparts [40]. However, it is imperative to take into account that the projection pattern of the CST, and thus the cortical control of upper limb movements, results from both direct and indirect corticomotoneuronal propriospinal transmission to motoneurons and can differ strikingly between primate species [5, 41].

In this study, we have set up a novel, reproducible model of partial injury of the spinal cord in M. murinus, a small NHP, and proceeded with a longitudinal follow-up over a 3-month period. Alterations in gross and fine motor function were quantifiable up to 2 weeks and throughout the experimental period, respectively. We chose a lateral hemisection of the spinal cord for many reasons; first, in humans the proportion of complete injury of the spinal cord is decreasing [42]; second, a lateral hemisection of the spinal cord permits a reproducible and precise control of lesion location and extent [12]; and third, an incomplete section permits preservation of critical physiological function (e.g., bladder and bowel functions) and thus reduces detrimental impacts on the health of the animal and the associated psychological trauma [5]. Lastly, as spared tissues play a critical role in local plasticity and inflammation, incomplete SCI allows us to uncover the underlying plastic mechanisms of recovery [11]. and provides a path for evaluating further therapeutic strategies to promote sprouting of spared axons [5]. Quadrupedalism in primates, particularly in M. murinus, is well adapted for walking on branches and differs from other mammals: they prefer a diagonal sequence to a lateral sequence [43]. Analysis of locomotion sequence in spinal cord injured primates walking quadrupedally had been done previously either on treadmill [13] or in a walking corridor [20]. The CatWalk system was originally designed for rodents that present a regularity index of $100 \%$ before lesion, but noninjured M. murinus scored $90 \%$, which we established as the "normal" value against which to compare functional recovery. We cannot exclude that the rather important functional recovery of gross motor activity that we observed following lateral spinal cord hemisection may partially result from bias introduced by CatWalk parameters analysis due to natural differences in the walking sequence between primates and nonprimates [43]. This may be particularly true for dynamic gait parameters (regularity index and duration of the swing phase); however, this rather important recovery is also consistent with a recent study demonstrating that primates exhibit better function recovery than rodents after lateralized SCI at the cervical level [6]. In that study, improved recovery correlated with greater reorganization of the CST rostral to the injury; indeed, monkeys, but not rats, presented growth/sprouting of corticospinal fibers across the spinal cord midline rostral to the injury. Monkeys, unlike rodents, present extensive bilateral CST projections and CST fibers are projected through both dorsolateral columns. Thus, as lateral spinal hemisection spares some CST fibers, further bilateral sprouting is possible and might underlie this rather substantial recovery $[6,8,9]$. In our study, the grip test allowed clear discrimination between hindlimbs ipsilateral and contralateral to the lesion up to 3 months postinjury. However, to further refine the accuracy of this test and better discriminate between different groups of M. murinus (e.g., treated $v s$ sham), video monitoring of the grip test may be necessary to allow better analysis of detailed toe movements. Also, adaptation of the "reach and grasp drawer" task, used for evaluating precise hand movements and grip force [44], may further increase the sensitivity of behavioral assessments. Indeed, the use of a force transducer on the bar would also allow quantitative assessments of exerted pressure and grip ability.

In parallel, using axial in vivo ${ }^{1} \mathrm{H}-\mathrm{MRI}$ acquisition for longitudinal follow-up, we studied lesion evolution over 3 months postinjury. Lesion extension was more pronounced caudal to the lesion epicenter than rostral, and the lesion volume peaked at 1 week postinjury, which may correspond to 
edema resorption. Owing to the absence of respiratory and cardiac motions and lack of time constraints, ex vivo ${ }^{1} \mathrm{H}$ MRI allows better resolution than in vivo ${ }^{1} \mathrm{H}-\mathrm{MRI}$. As in our previous study using mice [31], comparison of lesion volume between in vivo and ex vivo ${ }^{1} \mathrm{H}-\mathrm{MRI}$ and histology showed a higher lesion estimation using in vivo than ex vivo, most likely reflecting differences between fixed and living tissues.

Three months after injury, glial reactivity in the gray matter was greater adjacent to the lesion than distal to the injury, as well as rostral versus caudal to the lesion. In the dorsal funiculus region ipsilateral to the lesion, we identified a pronounced increase in specific microglia/macrophage reactivity rostral but not caudal to the lesion site. Conversely, astrocytes did not show increased reactivity within the dorsal funiculus. Ex vivo and in vivo ${ }^{1} \mathrm{H}-\mathrm{MRI}$ hypersignals coincided with the presence of activated microglia/macrophages within the dorsal funiculus. Identification of this hypersignal may thus, directly or most likely indirectly, represent a ${ }^{1} \mathrm{H}$-MRI signature of microglia/macrophage activation distal to the lesion epicenter. Indeed, blood-spinal cord barrier breakdown, as well as water content and permeability of blood vessels, are usually accompanied by microglia/macrophage activation and peripheral infiltration. A previous report in rodents also found a close correlation between activated microglia/macrophages within the lesion epicenter and MRI signal, suggesting that the T2weighted MRI signals are useful for localizing glial scarring and inflammation in the injured spinal cord [45]. The specific identification of microglia/macrophage activation and infiltration in the dorsal funiculus at the lesion site and rostral (but not caudal) to the lesion reflects differential response of both dorsal column nuclei and ascending spinal afferents. In mice, greater microglia/macrophage activation is also observed rostral but not caudal to the lesion within the entire dorsal funiculus (Perrin, personal communication). However, specific microglia/macrophage activation in the dorsal funiculus rostral and ipsilateral to the lesion might also reflect more complex anatomic specificities and injury-induced reorganization of the dorsal column nuclei in primates [46].

The "ideal" criteria for NHP models of SCI had been defined as 1) to present similar anatomic features; 2) to recapitulate the clinical pathology; 3 ) to be repeatable; and finally 4) to be available [11]. Knowing that such an animal model does not exist, development of new animal models will thus certainly contribute and complement our understanding of the basic biology that underlie SCI pathogenesis and conceivably lead to novel therapeutic strategies [47]. The neuroanatomical organization and glial distribution/morphology in M. murinus and human spinal cords present strong similarities, confirming the advantage of NHPs has a model of SCI. Lateral hemisection at the higher lumbar level of the spinal cord in M. murinus caused impairments in motor function of the ipsilateral hindlimb that can be quantitatively assessed at multiple time points following lesion. The small size of $M$. murinus and its resistance to repetitive anesthetic procedures allows reliable longitudinal follow-up of lesion evolution using an in vivo ${ }^{1} \mathrm{H}$-MRI apparatus initially designed for rodents. Interestingly, activated microglia/macrophages observed ipsilateral and rostral to the lesion epicenter in the dorsal funiculus coincide with ${ }^{1} \mathrm{H}$-MRI signals.

Thus, M. murinus is a reliable NHP model of lateral hemisection that can be used to complement other NHP models and extend translational research on SCI. It also represents and affordable alternative to larger NHPs for preclinical studies.

Required Author Forms Disclosure forms provided by the authors are available with the online version of this article.

\section{Compliance with Ethical Standards}

Competing Interests The authors declare that they have no competing interests. The authors declare no conflict of interest or competing financial interests. This work was supported by the patient organizations "Verticale" (to H.N.N., G.P.S.M., and F.E.P.) and "Demain Debout Aquitaine" (to H.N.N. and F.E.P.) and the LabeX NUMEV (to G.P.S.M.). Funding bodies had no role in the design of the study, analysis, and interpretation of data, or in the writing of the manuscript. We thank A. Privat for fruitful discussions. We also thank M. Cardoso for her technical assistance in acquisition of magnetic resonance imaging, P. Villette for his help in CatWalk analysis, S. Rouland for her help in animal handling, and A. Ciston for English-lanugage editing.

\section{References}

1. Sekhon LH, Fehlings MG. Epidemiology, demographics, and pathophysiology of acute spinal cord injury. Spine 2001;26(24 Suppl): S2-12.

2. Wyndaele M, Wyndaele JJ. Incidence, prevalence and epidemiology of spinal cord injury: what learns a worldwide literature survey? Spinal Cord 2006;44(9):523-529.

3. Ahuja CS, Wilson JR, Nori S, et al. Traumatic spinal cord injury. Nat Rev Dis Primers 2017;3:17018.

4. Buss A, Pech K, Kakulas BA, et al. NG2 and phosphacan are present in the astroglial scar after human traumatic spinal cord injury. BMC Neurol 2009;9:32.

5. Courtine G, Bunge MB, Fawcett JW, et al. Can experiments in nonhuman primates expedite the translation of treatments for spinal cord injury in humans? Nat Med 2007;13(5):561-566.

6. Friedli L, Rosenzweig ES, Barraud Q, et al. Pronounced species divergence in corticospinal tract reorganization and functional recovery after lateralized spinal cord injury favors primates. Sci Transl Med 2015;7(302):302ra134.

7. Lemon R. Cortico-motoneuronal system and dexterous finger movements. J Neurophysiol 2004;92(6):3601.

8. Lemon RN. Descending pathways in motor control. Annu Rev Neurosci 2008;31:195-218.

9. Lemon RN, Griffiths J. Comparing the function of the corticospinal system in different species: organizational differences for motor specialization? Muscle Nerve 2005;32(3):261-279.

10. Curt A, Van Hedel HJ, Klaus D, Dietz V. Recovery from a spinal cord injury: significance of compensation, neural plasticity, and repair. J Neurotrauma 2008;25(6):677-685. 
11. Nardone R, Florea C, Holler Y, et al. Rodent, large animal and nonhuman primate models of spinal cord injury. Zoology (Jena) 2017;123:101-114.

12. Nout YS, Rosenzweig ES, Brock JH, et al. Animal models of neurologic disorders: a nonhuman primate model of spinal cord injury. Neurotherapeutics 2012;9(2):380-392.

13. Rosenzweig ES, Courtine G, Jindrich DL, et al. Extensive spontaneous plasticity of corticospinal projections after primate spinal cord injury. Nat Neurosci 2010;13(12):1505-1510.

14. Cregg JM, DePaul MA, Filous AR, Lang BT, Tran A, Silver J. Functional regeneration beyond the glial scar. Exp Neurol 2014;253:197-207.

15. Buss A, Pech K, Kakulas BA, et al. Growth-modulating molecules are associated with invading Schwann cells and not astrocytes in human traumatic spinal cord injury. Brain 2007;130(Pt 4):940-953.

16. Shi F, Zhu H, Yang $\mathrm{S}$, et al. Glial response and myelin clearance in areas of wallerian degeneration after spinal cord hemisection in the monkey Macaca fascicularis. J Neurotrauma 2009;26(11):20832096.

17. Nishimura S, Sasaki T, Shimizu A, et al. Global gene expression analysis following spinal cord injury in non-human primates. Exp Neurol 2014;261:171-179.

18. Miller AD, Westmoreland SV, Evangelous NR, Graham A, Sledge J, Nesathurai S. Acute traumatic spinal cord injury induces glial activation in the cynomolgus macaque (Macaca fascicularis). $\mathrm{J}$ Med Primatol 2012;41(3):202-209.

19. Wu W, Jian Z, Shi F, et al. Axonal and glial responses to a midthoracic spinal cord hemisection in the Macaca fascicularis monkey. J Neurotrauma 2013;30:826-839.

20. Ma Z, Zhang YP, Liu W, et al. A controlled spinal cord contusion for the rhesus macaque monkey. Exp Neurol 2016;279:261-273.

21. Fischer KE, Austad SN. The development of small primate models for aging research. ILAR J 2011;52(1):78-88.

22. Orsi A, Rees D, Andreini I, Venturella S, Cinelli S, Oberto G. Overview of the marmoset as a model in nonclinical development of pharmaceutical products. Regul Toxicol Pharmacol 2011;59(1): 19-27.

23. Dupinay $\mathrm{T}$, Gheit $\mathrm{T}$, Roques $\mathrm{P}$, et al. Discovery of naturally occurring transmissible chronic hepatitis B virus infection among Macaca fascicularis from Mauritius Island. Hepatology 2013;58(5):1610-1620.

24. Carlson CS, O'Sullivan MG, Jayo MJ, et al. Fatal disseminated cercopithecine herpesvirus 1 herpes B infection in cynomolgus monkeys (Macaca fascicularis). Vet Pathol 1997;34(5):405-414.

25. Costa EA, Luppi MM, Malta Mde C, et al. Outbreak of human herpesvirus type 1 infection in nonhuman primates (Callithrix penincillata). J Wildlife Dis 2011;47(3):690-693.

26. Fechner K, Matz-Rensing K, Lampe K, Kaup FJ, Czerny CP, Schafer J. Detection of Mycobacterium avium subsp. paratuberculosis in non-human primates. J Med Primatol 2017;46(5):211-217.

27. Panarella ML, Bimes RS. A naturally occurring outbreak of tuberculosis in a group of imported cynomolgus monkeys (Macaca fascicularis). J Am Assoc Lab Anim Sci 2010;49(2):221-225.

28. Languille $\mathrm{S}, \mathrm{Blanc} \mathrm{S}, \mathrm{Blin} \mathrm{O}$, et al. The grey mouse lemur: a nonhuman primate model for ageing studies. Ageing Res Rev 2012;11(1):150-162.
29. Perret M. Relationship between urinary estrogen levels before conception and sex ratio at birth in a primate, the gray mouse lemur. Hum Reprod 2005;20(6):1504-1510.

30. Noristani HN, Boukhaddaoui H, Saint-Martin GP, et al. Microglial alterations after spinal cord injury: a combination of ex vivo diffusion MRI and multiphoton study. Front Aging Neurosci 2017;9: 230.

31. Noristani HN, Lonjon N, Cardoso M, et al. Correlation of in vivo and ex vivo (1)H-MRI with histology in two severities of mouse spinal cord injury. Front Neuroanat 2015;9:24.

32. Lonjon N, Kouyoumdjian P, Prieto M, et al. Early functional outcomes and histological analysis after spinal cord compression injury in rats. J Neurosurg Spine 2010;12(1):106-113.

33. Lonjon $\mathrm{N}$, Prieto $\mathrm{M}$, Haton $\mathrm{H}$, et al. Minimum information about animal experiments: supplier is also important. J Neurosci Res 2009;87(2):403-407.

34. Coillot C, Sidiboulenouar R, Nativel E, et al. Signal modeling of a MRI ribbon solenoid coil dedicated to spinal cord injuries investigations. J Sensors Sensor Syst 2016;5:137-45.

35. Noristani HN, Sabourin JC, Boukhaddaoui H, Chan-Seng E, Gerber YN, Perrin FE. Spinal cord injury induces astroglial conversion towards neuronal lineage. Mol Neurodegener 2016;11(1): 68.

36. Karnovsky MJ, Roots L. A "direct-coloring" thiocholine method for cholinesterases. J Histochem Cytochem 1964;12:219-221.

37. Muller F, O'Rahilly R. The early development of the nervous system in staged insectivore and primate embryos. J Comp Neurol 1980;193(3):741-751.

38. Zhang K, Sejnowski TJ. A universal scaling law between gray matter and white matter of cerebral cortex. Proc Natl Acad Sci U S A 2000;97(10):5621-5626.

39. Herculano-Houzel S. The glia/neuron ratio: how it varies uniformly across brain structures and species and what that means for brain physiology and evolution. Glia 2014;62(9):1377-1391.

40. Oberheim NA, Takano T, Han X, et al. Uniquely hominid features of adult human astrocytes. J Neurosci 2009;29(10):3276-3287.

41. Nakajima K, Maier MA, Kirkwood PA, Lemon RN. Striking differences in transmission of corticospinal excitation to upper limb motoneurons in two primate species. J Neurophysiol 2000;84(2): 698-709.

42. Devivo MJ. Epidemiology of traumatic spinal cord injury: trends and future implications. Spinal Cord 2012;50(5):365-372.

43. Shapiro LJ, Cole WG, Young JW, Raichlen DA, Robinson SR, Adolph KE. Human quadrupeds, primate quadrupedalism, and Uner Tan Syndrome. PLOS ONE 2014;9(7):e101758.

44. Schmidlin E, Kaeser M, Gindrat AD, et al. Behavioral assessment of manual dexterity in non-human primates. J Vis Exp 2011;57: 3258.

45. Byrnes KR, Fricke ST, Faden AI. Neuropathological differences between rats and mice after spinal cord injury. J Magn Reson Imaging 2010;32(4):836-846.

46. Rustioni A, Hayes NL, O'Neill S. Dorsal column nuclei and ascending spinal afferents in macaques. Brain 1979;102(1):95-125.

47. Peter AK, Crocini C, Leinwand LA. Expanding our scientific horizons: utilization of unique model organisms in biological research. EMBO J 2017;36(16):2311-2314. 